\title{
Scheduling When You Do Not Know the Number of Machines
}

\author{
CLIFFORD STEIN, Columbia University, USA \\ MINGXIAN ZHONG, Lehman College and the Graduate Center, City University of New York, USA
}

\begin{abstract}
Often in a scheduling problem, there is uncertainty about the jobs to be processed. The issue of uncertainty regarding the machines has been much less studied. In this article, we study a scheduling environment in which jobs first need to be grouped into some sets before the number of machines is known, and then the sets need to be scheduled on machines without being separated. To evaluate algorithms in such an environment, we introduce the idea of an $\alpha$-robust algorithm, one that is guaranteed to return a schedule on any number $m$ of machines that is within an $\alpha$ factor of the optimal schedule on $m$ machine, where the optimum is not subject to the restriction that the sets cannot be separated. Under such environment, we give a $\left(\frac{5}{3}+\epsilon\right)$ robust algorithm for scheduling on parallel machines to minimize makespan and show a lower bound $\frac{4}{3}$. For the special case when the jobs are infinitesimal, we give a 1.233-robust algorithm with an asymptotic lower bound of 1.207 . We also study a case of fair allocation, where the objective is to minimize the difference between the maximum and minimum machine load.
\end{abstract}

CCS Concepts: • Theory of computation $\rightarrow$ Scheduling algorithms; Adversary models;

Additional Key Words and Phrases: Robust algorithms

ACM Reference format:

Clifford Stein and Mingxian Zhong. 2019. Scheduling When You Do Not Know the Number of Machines. ACM Trans. Algorithms 16, 1, Article 9 (November 2019), 20 pages.

https://doi.org/10.1145/3340320

\section{INTRODUCTION}

For many problems, one does not know the entire input accurately and completely in advance. There are different ways of addressing such uncertainty, e.g., via online algorithms (assuming the input arrives over time), dynamic algorithms (assuming the input changes over time), stochastic optimization (assuming the input includes random variables), or robust optimization (assuming that there is bounded uncertainty in the data). Another way of addressing uncertainty is to require one solution that is good against all possible values of the uncertain parameters. Examples of work in this direction include the universal traveling salesman problem (one tour that is good no matter which subset of points arrive) [14], robust matchings (one matching is chosen and then evaluated by its top $k$ edges, where $k$ is unknown) $[9,11]$, a knapsack of unknown capacity (one policy

Our work is supported in part by NSF Grants No. CCF-1421161 and No. CCF-1714818.

Authors' addresses: C. Stein, Columbia University, 500 West 120th Street, New York, NY, 10027, USA; email: cliff@ieor. columbia.edu; M.Zhong, Lehman College and the Graduate Center, City University of New York, 250 Bedford Park Blvd W, Bronx, NY 10468, USA; email: mingxian.zhong@lehman.cuny.edu.

Permission to make digital or hard copies of all or part of this work for personal or classroom use is granted without fee provided that copies are not made or distributed for profit or commercial advantage and that copies bear this notice and the full citation on the first page. Copyrights for components of this work owned by others than ACM must be honored. Abstracting with credit is permitted. To copy otherwise, or republish, to post on servers or to redistribute to lists, requires prior specific permission and/or a fee. Request permissions from permissions@acm.org.

(C) 2019 Association for Computing Machinery.

1549-6325/2019/11-ART9 \$15.00

https://doi.org/10.1145/3340320 
of packing that is good irrespective of the actual capacity) [6], and two-stage scheduling (some decisions must be made before the actual scenario is known) [5, 17]. In scheduling problems, there are many ways to model uncertainty in the jobs, including online algorithms [1,2], in which the set of jobs is not known in advance, stochastic scheduling [13], in which the jobs are modeled as random variables, and work on schedules that are good against multiple objective functions $[4,12$, 15]. But there is much less work studying the possibility of uncertainty in the machines, and the work we are aware of studies uncertainty in speed or reliability (breakdowns) [3,7].

Motivated by the need to understand how to make scheduling decisions without knowing how many machines we will have, we consider a different notion of uncertainty-a scenario in which you don't know how many machines you are going to have, but you still have to commit (partially) to a schedule by making significant decisions about partitioning the jobs before knowing the number of machines.

This type of decision arises in a variety of settings. For example, many scheduling problems are fundamentally about packing items onto machines, and there are many examples of problems that concern packing items where there are multiple levels of commitment to be made with partial information. For example, in a warehouse, a large order may need to be placed into multiple boxes, without knowing exactly how many trucks there will be to ship the items. You therefore want to be able to pack the items well, given the various possible number of trucks. Another example involves problems in modern data centers. In data centers, there are some systems that require you to group work together into "bundles" without knowing exactly how many machines will be available. For example, in a map-reduce type computation, the mapping function naturally breaks the data into some number of groups $g$. However, there are some unknown number of available machines $m$, and you typically have to design your mapping function, choosing a $g$ and associated grouping, without knowing $m$. You may know a range of possible values for $m$, or it may vary widely depending on the availability of machines at the time you run the map-reduce computation (and the availability is typically not under your control). As more and more computing moves to the "cloud," that is, moves to large shared data centers, we anticipate that this problem of grouping work without knowing the number of machines will become more widespread.

In this article, we consider the following model. We are given a set of $n$ jobs, $J$, with a known processing times $p(j)$ for each job $j$, and a number $M$, which is an upper bound on the number of machines we might have. An algorithm must commit, before knowing how many machines there are, to grouping the jobs into $M$ bags, where each job is assigned to exactly one of the bags. We call this step the packing step. Only after completing the packing step do we learn the number of machines $m$. We now need to compute a schedule, with the restriction that we must keep the bags together, that is, we will assign one or more bags to each machine. We call this step the scheduling step. As in other robust problems, we want to do well against all possible numbers of machines. We therefore evaluate our schedule by the ratio of the makespan of our schedule, $A L G(m, M)$, to the makespan of a schedule that knew $m$ in advance, opt ${ }_{m}$, taking the worst case over all possible values of $m$. If an algorithm always provides a ratio of at most $\alpha$, where $\alpha=\max _{1 \leq m \leq M} \frac{A L G(m, M)}{\mathrm{opt}_{m}}$, then we call it $\alpha$-robust. (We may also consider scenarios in which there are different upper and lower bounds on the range of $m$; the definition of robustness extends in the obvious way.)

Our main result is an algorithm, which is $\left(\frac{5}{3}+\epsilon\right)$-robust, for minimizing makespan on parallel machines; and we show a lower bound of $4 / 3$ on the robustness of any algorithm for minimizing makespan on parallel machines. As with many scheduling problems, there are two different aspects to address. One is the load-balancing aspect, but in this two-stage problem, it seems that one wants to create bags of a variety of different sizes, to allow a more balanced final schedule. The second is to deal with large jobs and to handle cases where one or several large jobs are the dominant term 
in the makespan. Large jobs seem to provide a particular challenge in this problem, and much of our algorithm and analysis are devoted to handling various cases involving large jobs.

To focus on the load balancing issues, we also consider the "continuous" case where we have a set of infinitesimal jobs. That is, in the packing stage, we simply need to divide our total load into $M$ bags. In traditional makespan scheduling, this case is trivial, we would just divide the load into $M$ equal pieces and achieve an optimal makespan. But in this two stage-problem, even the continuous case is challenging. We can, however, obtain significantly stronger results than in the discrete case, showing an upper bound of 1.233 and a lower bound of 1.207 on the robustness. It is worth noting that in Reference [16], the author considered a similar model of sequential partitioning on an interval with the objective of keeping the largest subinterval small at all the stages of the partition process.

The continuous case also models a problem in fair allocation. In fair allocation, you typically have resources that you want to split "fairly" among several parties. The literature on this problem is vast, and we will not attempt to summarize it here. We will only observe that we are solving a problem in fair allocation that has not previously been studied, to our knowledge. We are given some objects to share, and everyone agrees on the values, but we do not know how many people will be sharing them. We place the objects into bags, and have the restriction that each person must take a subset of the bags. In the makespan variant, we are minimizing the maximum amount that anyone gets. Motivated by fairness, we also consider a version where you want to minimize the difference between the maximum allocation and the minimum allocation (this objective makes sense in fair allocation, but not necessarily in scheduling). Here, we consider a case where we know a lower bound of $\alpha M$ on the eventual number of machines, and can show a lower bound of $\min \{2 / 3,2 /(4 \alpha+1)\} A$ on the difference, and we can obtain an upper bound of $\min \{2 / 3,1 /(\alpha+1)\} A$, where $A$ is the average load.

\subsection{Overview of this Chapter and a Lower Bound}

We give a brief overview of this chapter, and for intuition, a simple lower bound. The order of our article is different than the order presented in the Introduction. We present the continuous case first, because the proofs are simpler and it gives some intuition for the discrete case.

In Section 2, we consider the case when all jobs are infinitesimal. We give an algorithm that is approximately 1.233 robust. And we also show a lower bound that is approximately 1.207 . For the infinitesimal case, we also consider the objective of minimizing the maximum difference between the most loaded and least loaded machine. For this case, and the average load is $A$, if we know that the eventual number of machines is in the range $[\alpha M, M]$, we can show an asymptotic lower bound of $\min \{2 / 3,2 /(4 \alpha+1)\} A$ on the difference, and we can obtain an upper bound of $\min \{2 / 3,1 /(\alpha+1)\} A$.

In Section 3, we consider the general case with arbitrary sized jobs. We give an algorithm that gives a robust ratio of $\frac{5}{3}+\epsilon$, breaking the simple 2 bound that can be obtained by running LPT on $M$ sets and then repeatedly merging the two smallest sets until $m$ sets remain. In our algorithm, we first calculate the optimal schedule for $m \in\left\{\frac{M}{2}, \frac{3 M}{4}, M\right\}$ within a factor of $1+\epsilon$ using the algorithm in Reference [10]. We then take one of these schedules and partition the jobs that were scheduled on some machines into a larger number of sets, which we call bags. After learning how many machines we have, we place the bags on the machines. This algorithm is more involved than the previous ones, and has several cases, based on the values of $\operatorname{opt}_{M}$, opt $\mathrm{op}_{3 M / 4}$, and opt $\mathrm{ol}_{M / 2}$ and demonstrates how a more careful investigation of the packing and scheduling steps can lead to improved bounds.

We conclude the Introduction with a simple lower bound. Consider the following example. Let the upper bound on the number of machines, $M$, be 3 and assume we have $n=6$ identical jobs, 


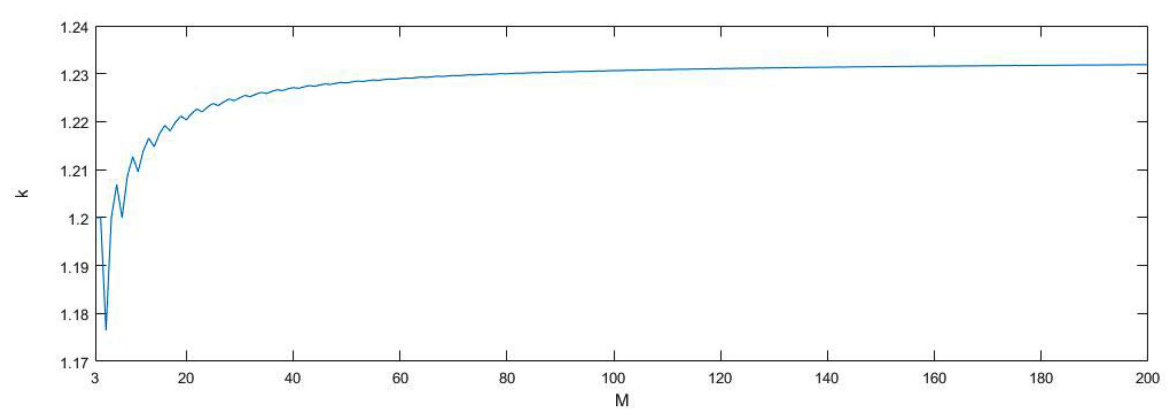

Fig. 1. $k$ as a function of $M$.

each of which has processing time 1 . We will prepare 3 bags that will ultimately have to be scheduled on either 1, 2, or 3 machines. Note that the unconstrained optimal makespan for 1, 2, and 3 machines are 6, 3, and 2, respectively. First, consider the packing that gives each bag 2 jobs. Then the makespans are 6, 4, and 2, respectively, if there are 1, 2, and 3 machines. Hence, this algorithm is $\max \{6 / 6,4 / 3,2 / 2\}=4 / 3$-robust. Next, consider the packing that places $\{1,2,3\}$ jobs in each bag, respectively. Then the makespans are 6,3 , and 3 , respectively, if there are 1,2 , and 3 machines, which makes this algorithm $\max \{6 / 6,3 / 3,3 / 2\}=3 / 2$-robust. In this example, the former algorithm is better. Moreover, this example demonstrates that $4 / 3$ is a lower bound on the robustness of any algorithm. Note that there exists a same lower bound for any number of $M$ : Consider an arbitrary $M$ and $n=2 M$ identical jobs with each processing time 1 , if we put at least one bag with at least 3 jobs, then the robust ratio is at least $3 / 2$; otherwise, we put 2 jobs in each bag and it provides a robust ratio that is at least $4 / 3$.

\section{SCHEDULING INFINITESIMAL JOBS}

Throughout this article, we use $p(j)$ to denote the processing time of job $j$. For any set of jobs $S$, we use $p(S)=\sum_{p_{i} \in S} p(i)$ to denote the sum of the processing times of jobs in $S$. We informally say a job set $S$ is big if the value of $p(S)$ is large and small otherwise.

We now consider the case of infinitesimal jobs. Suppose we are given a job set $J$ with all infinitesimal jobs such that $p(J)=s$, for some $s>0$. We first pack the jobs to $M \geq 3$ sets and then schedule the bags on $m$ machines, where $m \in\{1,2, \ldots, M\}$ is only known after we pack the jobs. Let $A L G(m, M)$ be the makespan of scheduling the bags on $m$ machines, then our objective is to minimize the robust ratio, $\alpha=\max _{m}\left\{\frac{A L G(m, M)}{\mathrm{opt}_{m}}\right\}$. Recall opt ${ }_{m}$ is the makespan of a schedule that knew $m$ in advance, and specifically opt ${ }_{m}=s / m$ when all jobs are infinitesimal.

The main idea in the packing is to produce a set of bags with a diverse set of sizes. More precisely, we consider the following packing, which we call packing PC. $S_{1}, S_{2}, \ldots, S_{M}$ are the bags: for $i=1,2,3, \ldots, 2\lfloor M / 3\rfloor, p\left(S_{i}\right)=\frac{k s}{M-\left\lceil\frac{i}{2}\right\rceil}-\frac{k s}{2(M-1)}$; for $j=2\lfloor M / 3\rfloor+1,2\lfloor M / 3\rfloor+2, \ldots, M$, $p\left(S_{j}\right)=\frac{k s}{M}$, where $k$ is a parameter that only depends on $M$, chosen to ensure that $\sum_{i=1}^{M} p\left(S_{i}\right)=s$. Specifically, $k=1 /\left(2 \cdot \sum_{i=1}^{\lfloor M / 3\rfloor} \frac{1}{M-i}-\left\lfloor\frac{M}{3}\right\rfloor \frac{1}{M(M-1)}+3\left\{\frac{M}{3}\right\} \frac{1}{M}\right) \approx 1.233$ when $M$ is large (here, we use \{\} to denote the integer remainder). And we will show that for all $M, k \leq 1.2333$. See Figure 1 to see how $k$ changes with $M$ and Figure 2 to see the size of bags when $M=100$ and $s=1000$.

Using packing PC to put the jobs into bags, we obtain the following theorem.

Theorem 2.1. For $m \in[1, M]$, there exists a schedule with makespan at most $k_{\text {opt }}{ }_{m} \leq 1.2333 \mathrm{opt}_{m}$, which schedules $\left\{S_{1}, S_{2}, \ldots, S_{M}\right\}$ on $m$ machines. 


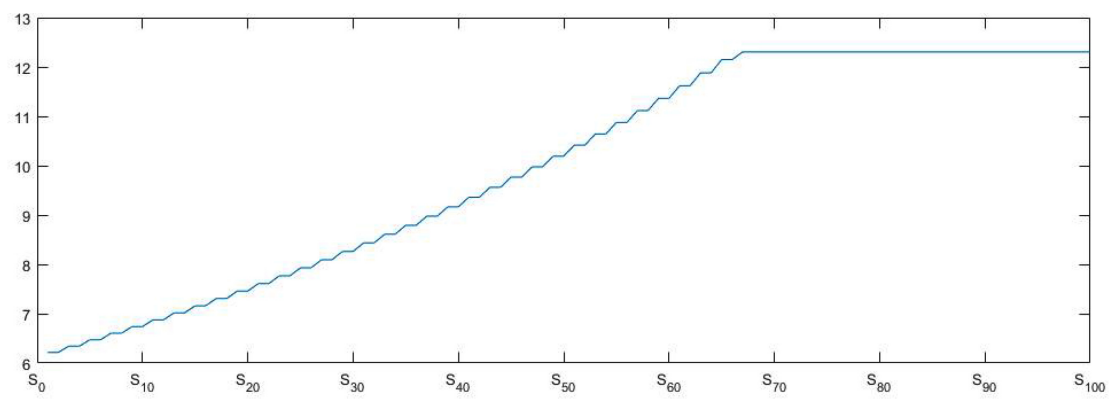

Fig. 2. The size of the bags when $M=100$ and $s=1000$.

Proof. We first consider the case when $m \geq M / 2$, and we schedule the bags as follows. Let $t=M-m$. For machine $i=1,2, \ldots, t$, we schedule bags $S_{i}$ and $S_{2 t-i+1}$ on machine $i$; for machine $j=t+1, \ldots, m$, we schedule bag $S_{j+t}$ on machine $i$. The machines with one bag are all within the bound, since for $i \leq 2\left\lfloor\frac{M}{3}\right\rfloor, p\left(S_{i}\right)=\frac{k s}{M-\left\lceil\frac{i}{2}\right\rceil}-\frac{k s}{2(M-1)} \leq \frac{k s}{M-M / 3}-\frac{k s}{2 M}=\frac{k s}{M} \leq \frac{k s}{m}=k$ opt $_{m}$. Therefore, it remains to bound the load on machines with two bags. We will use $L(i, t)$ to denote the load on the machine $i$ for a particular value of $t$. We therefore need to prove that $L(i, t)=p\left(S_{i}\right)+p\left(S_{2 t-i+1}\right) \leq k \operatorname{opt}_{m}$ for $1 \leq i \leq t, t=M-m \leq\left\lfloor\frac{M}{2}\right\rfloor$. Observe that when $i$ is even, $L(i, t)=p\left(S_{i}\right)+p\left(S_{2 t-i+1}\right)=p\left(S_{i-1}\right)+p\left(S_{2 t-i+2}\right)=L(i-1, t)$, hence we may assume that $i$ is odd. Since $i \leq\left\lfloor\frac{M}{2}\right\rfloor \leq 2\left\lfloor\frac{M}{3}\right\rfloor, p\left(S_{i}\right)=\frac{k s}{M-\frac{i+1}{2}}-\frac{k s}{2(M-1)}$.

We first consider the subcase when $2 t-i+1 \geq 2\left\lfloor\frac{M}{3}\right\rfloor+1$, then $p\left(S_{2 t-i+1}\right)=\frac{k s}{M}$. Since $i$ is odd, $i \leq 2 t-2\left\lfloor\frac{M}{3}\right\rfloor-1$. Hence, we have

$$
\begin{aligned}
L(i, t) & =\frac{k s}{M-\frac{i+1}{2}}-\frac{k s}{2(M-1)}+\frac{k s}{M} \\
& \leq \frac{k s}{M-t+\lfloor M / 3\rfloor}+\frac{k s(M-2)}{2(M-1) M} .
\end{aligned}
$$
that

Recall that opt $_{m}=\frac{k s}{m}=\frac{k s}{M-t}$. Since $t \geq\left\lfloor\frac{M}{3}\right\rfloor+\frac{i}{2}, t \geq\left\lfloor\frac{M}{3}\right\rfloor+1$. Using Equation (1), it follows

$$
\begin{aligned}
& L(i, t)-\frac{k s}{M-t} \\
& =\frac{k s}{M+\lfloor M / 3\rfloor-t}+\frac{k s(M-2)}{2(M-1) M}-\frac{k s}{M-t} \\
& \leq \frac{k s(M-2)}{2(M-1) M}-\frac{k s\lfloor M / 3\rfloor}{(M+\lfloor M / 3\rfloor-t)(M-t)} \\
& \leq \frac{k s(M-2)}{2(M-1) M}-\frac{k s\lfloor M / 3\rfloor}{(M-1)(M-\lfloor M / 3\rfloor-1)} \\
& =k s \frac{M^{2}-3 M+2-(3 M-2)\lfloor M / 3\rfloor}{2 M(M-1)(M-\lfloor M / 3\rfloor-1)} \\
& \leq k s \frac{M^{2}-3 M+2-(3 M-2)\left(M / 3-\frac{2}{3}\right)}{2 M(M-1)(M-\lfloor M / 3\rfloor-1)} \\
& =k s \frac{-M / 3+\frac{2}{3}}{2 M(M-1)(M-\lfloor M / 3\rfloor-1)}<0 .
\end{aligned}
$$


That is, $L(i, t) \leq \frac{k s}{M-t}=k \mathrm{opt}_{m}$. Next, we consider the subcase that that $2 t-i+1 \leq 2\left\lfloor\frac{M}{3}\right\rfloor$. Now, we have $p\left(S_{2 t-i+1}\right)=\frac{k s}{M-\frac{2 t-i+1}{2}}-\frac{k s}{2(M-1)}=\frac{k s}{M+(i+1) / 2-(t+1)}-\frac{k s}{2(M-1)}$ (Recall that we assume $i$ is odd then $2 t-i+1$ is even). Hence, we have

$$
\begin{aligned}
L(i, t) & =\frac{k s}{M-\frac{i+1}{2}}+\frac{k s}{M+\frac{i+1}{2}-(t+1)}-\frac{k s}{M-1} \\
& =\frac{k s(2 M-t-1)}{\left(M-\frac{i+1}{2}\right)\left(M+\frac{i+1}{2}-(t+1)\right)}-\frac{k s}{M-1} \\
& =\frac{k s(2 M-t-1)}{-\frac{1}{4}(i-t)^{2}+\frac{1}{4}(t+1)^{2}+M^{2}-(t+1) M}-\frac{k s}{M-1} \\
& \leq \frac{k s(2 M-t-1)}{-\frac{1}{4}(1-t)^{2}+\frac{1}{4}(t+1)^{2}+M^{2}-(t+1) M}-\frac{k s}{M-1} \\
& =\frac{k s(2 M-t-1)}{(M-1)(M-t)}-\frac{k s}{M-1}=\frac{k s}{M-t} .
\end{aligned}
$$

The inequality holds, because $1 \leq i \leq t$. This proves that for the case $m \geq M / 2$, the makespan of our schedule is at most $\frac{k s}{M-t}=k \mathrm{opt}_{m}$.

Next, we consider the case when $1 \leq m<M / 2$. Let $x \geq 2$ be the integer such that $\frac{M}{x+1} \leq m<\frac{M}{x}$. Let $t=M-m x$. For machine $i=1,2, \ldots, t$, we schedule bags $S_{i}, S_{2 t-i+1}$, $S_{i+2 t}, \ldots, S_{i+x t}$ on such machine; for machine $j=t+1, \ldots, m$, we schedule bags $S_{j+x t}, S_{j+x t+(m-t)}$, $S_{j+x t+2(m-t)} \ldots, S_{j+x t+(x-1)(m-t)}$ on such machine. Recall that $\forall i \leq M, p\left(S_{i}\right) \leq \frac{k s}{M}$. Observe that we schedule $x$ bags on the last $m-t$ machines, hence the processing times of jobs on such those machines are at most $\frac{x k s}{M}=\frac{k s}{M / x} \leq \frac{k s}{m}=k \mathrm{opt}_{m}$. The processing time of the bags scheduled on machine $i \leq t$ is (note that $m=(M-t) / x$ ):

$$
\begin{aligned}
p\left(S_{i}\right)+p\left(S_{2 t-i+1}\right)+\sum_{j=2}^{x} p\left(S_{i+j t}\right) & =L(i, t)+\sum_{j=2}^{x} p\left(S_{i+j t}\right) \\
& \leq \frac{k s}{M-t}+(x-1) \frac{k s}{M} \\
& \leq \frac{k x s}{M-t}=\text { opt }_{m} .
\end{aligned}
$$

The last is to show a bound of $k$. Recall that $k$ is chosen to ensure that $\sum_{i=1}^{M} p\left(S_{i}\right)=s$, so we have (recall that we use \{\} to denote the integer remainder)

$$
\begin{aligned}
\sum_{i=1}^{M} p\left(S_{i}\right) & =2 \cdot \sum_{i=1}^{\lfloor M / 3\rfloor}\left(\frac{k s}{M-i}-\frac{k s}{2(M-1)}\right)+(M-2\lfloor M / 3\rfloor) \cdot \frac{k s}{M} \\
& =2 \cdot \sum_{i=1}^{\lfloor M / 3\rfloor} \frac{k s}{M-i}-\lfloor M / 3\rfloor \frac{k s}{M(M-1)}+3\{M / 3\} \frac{k s}{M} .
\end{aligned}
$$

We can solve Equation (2) for $k$ and obtain that $k=1 / b(M)$, where $b(M)=2 \cdot \sum_{i=1}^{\lfloor M / 3\rfloor} \frac{1}{M-i}-$ $\left\lfloor\frac{M}{3}\right\rfloor \frac{1}{M(M-1)}+3\left\{\frac{M}{3}\right\} \frac{1}{M}$. Note that when $M$ is large,

$$
\begin{aligned}
b(M) & \approx 2 \sum_{i=M-\lfloor M / 3\rfloor}^{M-1} \frac{1}{i} \approx 2(\ln (M-1)-\ln (2 M / 3)) . \\
& \approx 2 \ln 1.5
\end{aligned}
$$


Hence, $k=1 / b(M) \approx 1 /(2 \ln 1.5) \approx 1.233$ when $M$ is large. We verify by computer that when $M<$ $10,000, k \leq 1.2333$. And for $M \geq 10,000$,

$$
\begin{aligned}
b(M) & \geq 2 \cdot \int_{0}^{\lfloor M / 3\rfloor} \frac{1}{M-i}-\frac{1}{3(M-1)} \\
& \geq 2 \ln \frac{M-\lfloor M / 3\rfloor}{M}-4 \cdot 10^{-5} \geq 0.81089 .
\end{aligned}
$$

So, $k \leq 1 / 0.81089 \leq 1.2333$.

We can also show a lower bound. That is, we can show that, no matter how you divide the jobs, you cannot achieve a robust ratio below 1.207, which is close to (but does not exactly match) our upper bound. We state the theorem in terms of a function $Q(M)$, which we define precisely below and which, for large $M$, is 1.207 .

Theorem 2.2. Let $S_{1}, S_{2}, \ldots, S_{M}$ be $M$ bags of infinitesimal jobs such that $\sum_{i=1}^{M} p\left(S_{i}\right)=s$ for some $s>0$. Assume there exists a constant $k^{\prime}$ such for all $m \in\{1,2, \ldots, M\}$, we can schedule $\left\{S_{1}, S_{2}, \ldots, S_{M}\right\}$ on $m$ machines with makespan at most $k^{\prime} o p t_{m}$. Then $k^{\prime} \geq Q(M)$, where $Q(M)=$ $\max _{t \leq \frac{M}{2}, t \in \mathbb{N}} \frac{1}{\frac{t}{M-t}+\frac{M-2 t}{M}} \approx 1.207$.

Proof. We may assume that $p\left(S_{1}\right) \leq p\left(S_{2}\right) \leq \cdots \leq p\left(S_{M}\right)$. We first prove the following stateme

Fort $\leq M / 2$, there exists a schedule of $\left\{S_{1}, S_{2}, \ldots, S_{M}\right\}$ on $m=M-t$ machines with minimum makespan such that $S_{1}, S_{2}, \ldots, S_{2 t}$ are on the first $t$ machines.

Let $\left\{T_{1}, T_{2}, \ldots, T_{m}\right\}$ be a schedule of $\left\{S_{1}, S_{2}, \ldots, S_{M}\right\}$ on $m$ machines with minimum makespan, where $T_{i}$ is a set of bags scheduled on machine $i$. We may assume that every $T_{i}$ contains at least one bag. Since we schedule $M$ bags on $M-t$ machines, there are at least $M-2 t$ machines contain exactly one bag. We rename the machines such that $T_{t+1}, T_{t+2}, \ldots, T_{t+(M-2 t)}=T_{m}$ contain only one bag. Suppose that at least one of $S_{1}, S_{2}, \ldots, S_{2 t}$ is not scheduled on the first $t$ machines, that is, there exists $i \leq 2 t, j \geq t+1$ such that $T_{j}=\left\{S_{i}\right\}$. Then, since $\left|\bigcup_{i=1}^{t} T_{i}\right|=M-\left|\bigcup_{i=t+1}^{m} T_{i}\right|=2 t$, there exists $i^{\prime} \geq 2 t+1, j^{\prime} \leq t$ such that $S_{i^{\prime}} \in T_{j^{\prime}}$. Define $T_{j}^{\prime}=\left\{S_{i^{\prime}}\right\}, T_{j^{\prime}}^{\prime}=T_{j^{\prime}} \backslash\left\{S_{i^{\prime}}\right\} \cup\left\{S_{i}\right\}$, and $T_{\ell}^{\prime}=T_{\ell}$ for $\ell \neq j, j^{\prime}$. Since $p\left(S_{i^{\prime}}\right) \geq p\left(S_{i}\right), p\left(T_{j^{\prime}}^{\prime}\right) \leq p\left(T_{j^{\prime}}\right)$. Note also that $p\left(T_{j}^{\prime}\right) \leq \max _{i=1}^{M}\left\{p\left(S_{i}\right)\right\} \leq$ $\max _{i=1}^{m}\left\{p\left(T_{i}\right)\right\}$. Hence, $\max _{i=1}^{m}\left\{p\left(T_{i}^{\prime}\right)\right\} \leq \max _{i=1}^{m}\left\{p\left(T_{i}\right)\right\}$. This implies that $\left\{T_{1}^{\prime}, \ldots, T_{m}^{\prime}\right\}$ is also a schedule with minimum makespan. Note that the first $t$ machines of schedule $\left\{T_{1}^{\prime}, \ldots, T_{m}^{\prime}\right\}$ contain more bags from $\left\{S_{1}, S_{2}, \ldots, S_{2 t}\right\}$ than the first $t$ machines of schedule $\left\{T_{1}, \ldots, T_{m}\right\}$. By repeating the above process, we can get a schedule with minimum makespan such that $S_{1}, S_{2}, \ldots, S_{2 t}$ are all scheduled on the first $t$ machines. This completes the proof of Equation (1).

By choosing $m=M$, we know that $p\left(S_{i}\right) \leq k^{\prime}$ opt $_{M}=\frac{k^{\prime} s}{M}$ for any $1 \leq i \leq M$. For $t \leq M / 2$, consider the schedule with minimum makespan on $m=M-t$ machines such that $S_{1}, S_{2}, \ldots, S_{2 t}$ are scheduled on the first $t$ machines. It follows that $\sum_{i=1}^{2 t} p\left(S_{i}\right) \leq t \cdot k^{\prime} \mathrm{opt}_{m}=\frac{t k^{\prime} s}{M-t}$

Hence, for any $t \leq M / 2$, we have

$$
\begin{aligned}
s=\sum_{i=1}^{M} p\left(S_{i}\right) & =\sum_{i=1}^{2 t} p\left(S_{i}\right)+\sum_{i=2 t+1}^{M} p\left(S_{i}\right) \\
& \leq \frac{t k^{\prime} s}{M-t}+(M-2 t) \cdot \frac{k^{\prime} s}{M} .
\end{aligned}
$$

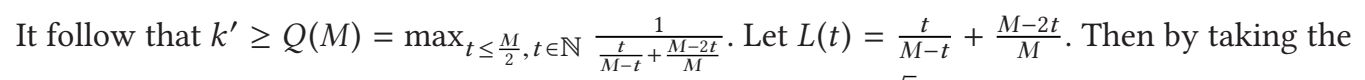
derivative, it is not hard to obtain that $\min _{0 \leq t \leq \frac{M}{2}} L(t)=L\left(\left(1-\frac{\sqrt{2}}{2}\right) M\right)=2(\sqrt{2}-1)$. And $Q(M) \approx$ $\frac{1}{\min _{0 \leq t \leq \frac{M}{2}} L(t)}=\frac{\sqrt{2}+1}{2} \approx 1.207$.

Figure 3 shows how $Q(M)$ changes with $M$. 


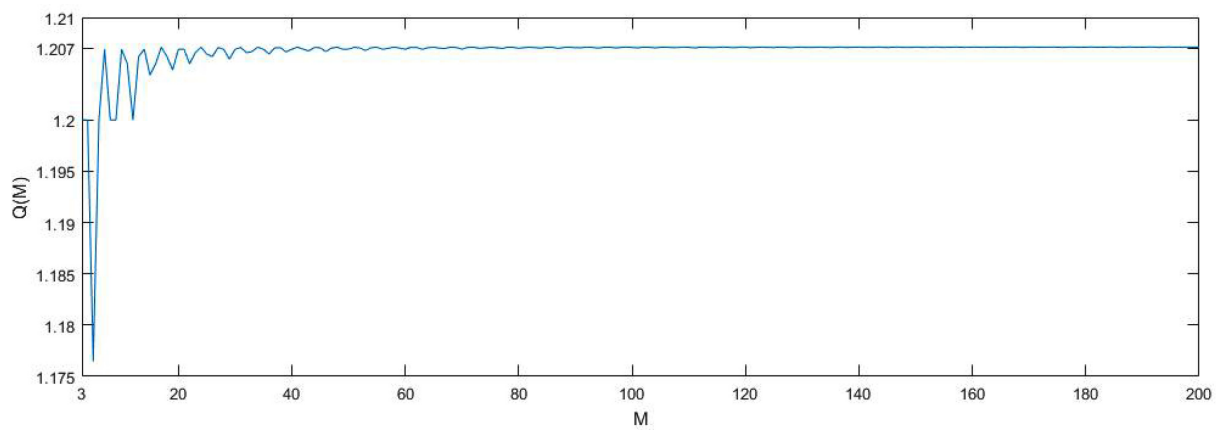

Fig. 3. $Q(M)$ as a function of $M$.

\subsection{Minimizing the Maximum Difference}

Another objective we consider is to minimize the difference between the load of the most loaded and least loaded machines. This objective is particularly relevant to settings in which we want to achieve fairness. First, minimizing the maximum difference is a well-studied scheduling objective in situations where fairness is important. Second, it models a type of fair allocation problem. Suppose that we want to split some goods among $m$ people, where we do not know what $m$ is in advance. To simplify the process, we can first divide the goods into $M$ groups or bags, and then have the restriction that each person must take a subset of the bags.

For this problem, we consider a case where after packing the jobs into $M$ bags, we are given $m \in[\alpha M, M]$ machines on which to schedule. Our bounds (see below) will depend on $\alpha$, with, not surprisingly, better bounds for larger $\alpha$. Since we assume every job is infinitesimal, the minimum difference between the load of the most loaded and least loaded machines is always 0 if we know the number of machines in advance. So, we cannot use the $\alpha$-robust settings to evaluate our algorithms in this section, and we introduce some new definitions here. Let $s$ denote the sum of processing time of all jobs and let $A=s / M$. For a set of bags $S=\left\{S_{1}, S_{2}, \ldots, S_{M}\right\}$, we use $D_{m}(M, \alpha, S, A)$ to denote the minimum difference between the load of the most loaded and least loaded machines when we schedule $S$ on $m$ machines. Our goal is to choose $S$ to minimize $D(M, \alpha, S, A)=\max _{m \in[\alpha M, M]}\left\{D_{m}(M, \alpha, S, A)\right\}$.

Assume that $\alpha M$ is an integer with value at most $M-1$ and $M \geq 3$. Let $D^{*}(M, \alpha, A)=$ $\min _{S}\{D(M, \alpha, S, A)\}$. We now give a lower bound of $D^{*}(M, \alpha, A)$.

THEorem 2.3. If $\alpha M>\frac{M}{2}$, then

$$
D^{*}(M, \alpha, A) \geq \frac{2(1-\alpha) M}{1+(4 \alpha+1)(1-\alpha) M} \cdot A .
$$

If $\alpha M \leq \frac{M}{2}$, then

$$
\begin{gathered}
D^{*}(M, \alpha, A) \geq \frac{2 M^{2}-2 M}{3 M^{2}+M-2} \cdot A, \text { if } M \text { is odd } \\
D^{*}(M, \alpha, A) \geq \frac{2 M^{2}-4 M}{3 M^{2}-8} \cdot A, \text { if } M \text { is even. }
\end{gathered}
$$

Proof. We may assume that $p\left(S_{1}\right) \leq p\left(S_{2}\right) \leq \cdots \leq p\left(S_{M}\right)$. For $i=1,2, \ldots, M$, let $A_{i}=$ $\sum_{j=1}^{i} p\left(S_{j}\right) / i$. We first prove the following statement:

$$
\text { For } m>M / 2, D_{m}(M, \alpha, S, A) \geq 2 A_{2 M-2 m}-p\left(S_{2 M-2 m+1}\right) \text {. }
$$


Assume the optimal way to schedule $\left\{S_{i}\right\}$ on $m$ machines is by scheduling a set of bags, $Y_{i}$, on the $i$ th machine. We may assume that every $Y_{i}$ contains at least one bag. Note that are at least $2 m-M$ machine receive exactly one bag. We rename the machines so that $Y_{M-m+1}, Y_{M-m+2}, \ldots, Y_{M-m+(2 m-M)}=Y_{m}$ contain only one bag. If the $2 m-M$ bags on those machines are exactly the biggest $2 m-M$ bags, $S_{2 M-2 m+1}, S_{2 M-2 m+2}, \ldots, S_{2 M-2 m+2 m-M}=S_{M}$, then $S_{1}, S_{2}, \ldots, S_{2 M-2 m}$ are on the first $M-m$ machines and it follow that the maximum load on a machines is at least $\sum_{i=1}^{2 M-2 m} S_{i} /(M-m)=2 A_{2 M-2 m}$ and the minimum load on a machine is at most $S_{2 M-2 m+1}$, which implies that (4) holds. So, we may assume not, and moreover among all optimal schedule, $\left\{Y_{i}\right\}$ is the schedule with the largest number of the biggest $2 m-M$ bags on the last $2 m-M$ machines. Then there exists $i \leq 2 M-2 m, j \geq M-m+1$ such that $Y_{j}=\left\{S_{i}\right\}$. It follow that there also exists $i^{\prime} \geq 2 M-2 m+1, j^{\prime} \leq M-m$ such that $S_{i^{\prime}} \in Y_{j^{\prime}}$. Define $Y_{j}^{\prime}=$ $\left\{S_{i^{\prime}}\right\}, Y_{j^{\prime}}^{\prime}=\left(Y_{j^{\prime}} \backslash\left\{S_{i^{\prime}}\right\}\right) \cup\left\{S_{i}\right\}$, and $Y_{\ell}^{\prime}=Y_{\ell}$ for $\ell \neq j, j^{\prime}$. Since $p\left(S_{i^{\prime}}\right) \geq p\left(S_{i}\right), p\left(Y_{j^{\prime}}^{\prime}\right) \geq p\left(Y_{j^{\prime}}\right)$. Note also that $p\left(Y_{j}^{\prime}\right) \leq \max _{i=1}^{M}\left\{p\left(S_{i}\right)\right\} \leq \max _{i=1}^{M}\left\{p\left(Y_{i}\right)\right\}$. Hence, $\max _{i=1}^{m}\left\{p\left(Y_{i}^{\prime}\right)\right\} \leq \max _{i=1}^{m}\left\{p\left(Y_{i}\right)\right\}$. Since $p\left(Y_{j^{\prime}}^{\prime}\right) \geq p\left(S_{i}\right)=p\left(Y_{j}\right)$ and $p\left(Y_{j}^{\prime}\right)=p\left(S_{i^{\prime}}\right) \geq p\left(Y_{j}\right), \min _{i=1}^{m}\left\{p\left(Y_{i}^{\prime}\right)\right\} \geq \min _{i=1}^{m}\left\{p\left(Y_{i}\right)\right\}$. This implies that $\left\{Y_{i}^{\prime}\right\}$ is also an optimal way of scheduling the bags. But in the last $2 m-M$ machines of $\left\{Y_{i}^{\prime}\right\}$, they have more bags come from the biggest $2 m-M$ bags than the last $2 m-M$ machines of $\left\{Y_{i}\right\}$, a contradiction. This completes the proof of Equation (4).

Let $D=D^{*}(M, \alpha, A)$ and let $S=\left\{S_{i}\right\}$ be the set of bags that reaches the optima. First, we assume that $\alpha M>\frac{M}{2}$. Let $A^{\prime}=A_{2 M-2 \alpha M}$. By Equation (4), $p\left(S_{(2-2 \alpha) M+1}\right) \geq 2 A^{\prime}-D_{\alpha M}(S) \geq 2 A^{\prime}-D$. Since $D \geq D_{M}(S)=p\left(S_{M}\right)-p\left(S_{1}\right) \geq p\left(S_{(2-2 \alpha) M+1}\right)-p\left(S_{1}\right)$, it follows that $p\left(S_{1}\right) \geq 2 A^{\prime}-2 D$ and $p\left(S_{M}\right) \geq p\left(S_{(2-2 \alpha) M+1}\right) \geq 2 A^{\prime}-D$.

We know that $A_{2} \geq p\left(S_{1}\right) \geq 2 A^{\prime}-2 D$. For $1 \leq k<(1-\alpha) M$, assume we know that $A_{2 k} \geq 2 A^{\prime}-$ $2 D+\frac{k-1}{2}\left(2 A^{\prime}-3 D\right)$. Then by Equation (4), we have $p\left(S_{2 k+1}\right) \geq 2 A_{2 k}-D_{M-k}(S) \geq 2\left(2 A^{\prime}-2 D+\right.$ $\left.\frac{k-1}{2}\left(2 A^{\prime}-3 D\right)\right)-D=2 A^{\prime}-2 D+k\left(2 A^{\prime}-3 D\right)$. Also, $p\left(S_{2 k+2}\right) \geq p\left(S_{2 k+1}\right) \geq 2 A^{\prime}-2 D+k\left(2 A^{\prime}-\right.$ $3 D)$. Therefore, $A_{2 k+2} \geq A_{2 k} \frac{2 k}{2 k+2}+\left(2 A^{\prime}-2 D+k\left(2 A^{\prime}-3 D\right)\right) \frac{2}{2 k+2} \geq 2 A^{\prime}-2 D+\frac{k}{2}\left(2 A^{\prime}-3 D\right)$. Inductively, this implies that $A^{\prime}=A_{2 M-2 \alpha M} \geq 2 A^{\prime}-2 D+\frac{(1-\alpha) M-1}{2}\left(2 A^{\prime}-3 D\right)$, which is equivalent to

$$
D \geq \frac{2(1-\alpha) M}{1+3(1-\alpha) M} A^{\prime}
$$

Recall that $p\left(S_{i}\right) \leq p\left(S_{1}\right)+D \leq A^{\prime}+D$ for any $i$, we have

$$
\begin{aligned}
M A=s & \leq 2(1-\alpha) M A^{\prime}+(2 \alpha-1) M\left(A^{\prime}+D\right) \\
& =M A^{\prime}+(2 \alpha-1) M D \\
& \leq \frac{1+3(1-\alpha) M}{2(1-\alpha)} D+(2 \alpha-1) M D .
\end{aligned}
$$

This is equivalent to

$$
D \geq \frac{2(1-\alpha) M}{1+(4 \alpha+1)(1-\alpha) M} \cdot A .
$$

For the case of $\alpha M \leq \frac{M}{2}$, if $M$ is odd, then

$$
D^{*}(M, \alpha, A) \geq D^{*}\left(M, \frac{M+1}{2 M}, A\right) \geq \frac{2 M^{2}-2 M}{3 M^{2}+M-2} \cdot A .
$$

If $M$ is even, then

$$
D^{*}(M, \alpha, A) \geq D^{*}\left(M, \frac{M+2}{2 M}, A\right) \geq \frac{2 M^{2}-4 M}{3 M^{2}-8} \cdot A .
$$


These bounds can most simply be parsed by saying that if $M$ is large, then the lower bound is given by $\min \{2 / 3,2 /(4 \alpha+1)\} A$. Note that when $\alpha \leq \frac{1}{2}$, the lower bound goes to $\frac{2}{3} A$ as $M$ goes large. If $M$ is even, then the bound of $\frac{2}{3} A$ can be reached by setting the bags as follows. Choose $S=$ $\left\{S_{1}, S_{2}, \ldots, S_{M}\right\}$ such that $p\left(S_{1}\right)=p\left(S_{2}\right)=\cdots=p\left(S_{M / 2}\right)=\frac{2}{3} A$ and $p\left(S_{M / 2+1}\right)=p\left(S_{M / 2+2}\right)=\cdots=$ $p\left(S_{M}\right)=\frac{4}{3} A$, then it is easy to verify that $D_{m}(S) \leq \frac{2}{3} A$ for any $m \in[1, M]$.

However, we can also get an upper bound of $D^{*}(M, \alpha, A)$ as follows when $\alpha>\frac{1}{2}$ (note that $D \approx \frac{1}{\alpha+1} \cdot A$ if $M$ is large).

THEOREM 2.4. If $\alpha M>\frac{M}{2}$, then

$$
D^{*}(M, \alpha, A) \leq \frac{(1-\alpha) M}{\alpha+(1-\alpha)(\alpha+1) M} \cdot A .
$$

Proof. Let $D=\frac{(1-\alpha) M}{\alpha+(1-\alpha)(\alpha+1) M} \cdot A \quad$ and $\quad A^{\prime}=\frac{1+3(1-\alpha) M}{2(1-\alpha) M} \cdot D$. We choose the bags, $T=\left\{T_{1}, T_{2}, \ldots, T_{M}\right\}$, as follows: for $k=0,1,2, \ldots,(1-\alpha) M-1$, set $p\left(T_{2 k+1}\right)=p\left(T_{2 k+2}\right)=$ $2 A^{\prime}-2 D+k\left(2 A^{\prime}-3 D\right)$; for $i \geq 2(1-\alpha) M+1$, set $p\left(T_{i}\right)=2 A^{\prime}-D$.

It is not hard to check that $\sum_{i=1}^{M} p\left(T_{i}\right)=M A$, and that the maximum difference of $p\left(T_{i}\right)$ is $D$. To show that $D^{*}(M, \alpha, A) \leq D$, it is sufficient to show that $D_{m}(M, \alpha, T, A) \leq D$ for any $m \in[\alpha M, M)$. Consider the following schedule on $m$ machines with $S_{i}$ being the set of bags on machine $i: S_{1}=$ $\left\{T_{1}, T_{2 M-2 m}\right\}, S_{2}=\left\{T_{2}, T_{2 M-2 m-1}\right\}, \ldots, S_{M-m}=\left\{T_{M-m}, T_{M-m+1}\right\}, S_{M-m+1}=\left\{T_{2 M-2 m+1}\right\}, S_{M-m+2}=$ $\left\{T_{2 M-2 m+2}\right\}, \ldots, S_{m}=\left\{T_{M}\right\}$. Then $\max _{i}\left\{p\left(S_{i}\right)\right\}=p\left(T_{1}\right)+p\left(T_{2 M-2 m}\right)=4 A^{\prime}-4 D+(M-m-1)$ $\left(2 A^{\prime}-3 D\right)$ and $\min _{i}\left\{p\left(S_{i}\right)\right\}=p\left(T_{2 M-2 m+1}\right)=2 A^{\prime}-2 D+(M-m)\left(2 A^{\prime}-3 D\right)$. So $D_{m}(M, \alpha, T, A) \leq$ $\left(4 A^{\prime}-4 D+(M-m-1)\left(2 A^{\prime}-3 D\right)\right)-\left(2 A^{\prime}-2 D+(M-m)\left(2 A^{\prime}-3 D\right)\right)=D$, as required.

It is worth remarking that another well-studied fairness objective function is to maximize the minimum loads of the machines. The analysis of this objective is easy in our model. Suppose we are given a job set $J$ with all infinitesimal jobs such that $p(J)=s$, for some $s>0$. We first pack the jobs to $M \geq 3$ sets, $\left\{S_{1}, S_{2}, \ldots, S_{M}\right\}$, and then schedule the bags on $m$ machines, where $m \in$ $\{1,2, \ldots, M\}$ is only known after we pack the jobs, such that the minimum load on the machines is maximized. Let $Y_{i}$ be the set of the bags on machine $i$ with $I_{m}=\min _{i} p\left(Y_{i}\right)$, then our objective is to deciding the size of the bags such that $R=\min _{m}\left\{I_{m} /(s / m)\right\}$ is maximized, where $s / m$ is the optimal minimum load. We may assume $p\left(S_{1}\right) \leq p\left(S_{2}\right) \leq \cdots \leq p\left(S_{M}\right)$. Consider the case when $m=M-k$ for $k=0,1, \ldots,\lceil M / 2\rceil-1$, then it is clear that $I_{M-k} \leq p\left(S_{2 k+1}\right)$, and then $p\left(S_{2 k+2}\right) \geq p\left(S_{2 k+1}\right) \geq$ $I_{m-k} \geq R \cdot s /(M-k)$. We assume $M$ is even for simplicity, then $s=\sum_{i=1}^{M} p\left(S_{i}\right) \geq 2 R s \sum_{k=0}^{M / 2-1} \frac{1}{M-k}$. It follows that $R \leq 1 /\left(2 \sum_{k=0}^{M / 2-1} \frac{1}{M-k}\right)=1 /\left(2 \sum_{j=M / 2+1}^{M} \frac{1}{j}\right) \approx 1 /(2 \ln 2) \approx 0.72$. And this upper bound can indeed be reached by taking $R=1 /\left(2 \sum_{k=0}^{M / 2-1} \frac{1}{M-k}\right)$ and $p\left(S_{2 k+2}\right)=p\left(S_{2 k+1}\right)=R \cdot s /(M-k)$ for $k=0,1, \ldots,\lceil M / 2\rceil-1$.

\section{SCHEDULING DISCRETE JOBS}

In this section, we will provide an algorithm that, for any set of jobs, gives a $\frac{5}{3}+\epsilon$ robust ratio for $m \in\{1,2, \ldots, M\}$. For ease of presentation, we first assume $M$ is divisible by 4 and prove Lemma 3.8. Then, we discuss the changes that are needed when $M$ is not divisible by 4 and prove Lemma 3.9. The two lemmas together gives the main theorem.

Our algorithm will start by computing minimum makespan schedules for $M, 3 M / 4$ and $M / 2$ machines. We can use a PTAS for minimizing makespan on parallel machines to compute a $(1+\epsilon)$ approximate schedule. We use opt ${ }_{i}^{\prime}$ to denote the value of the makespan obtained by running the PTAS on a scheduling instance with $i$ machines. We will especially use $\operatorname{opt}_{M / 2}^{\prime}$ and will denote this value by $b$. We use PTAS(i) $=\left\{S_{1}, S_{2}, . . S_{i}\right\}$ to denote the result of the PTAS on a scheduling instance with $i$ machines, where $S_{j}$ is the set of jobs assigned to machine $j$. 
Based on the values opt ${ }_{M}^{\prime}$, opt ${ }_{3 M / 4}^{\prime}$ and opt ${ }_{M / 2}^{\prime}$, we will consider several cases. Each case will have the same structure. First, we will compute sets of jobs $S_{i}$ from a PTAS. Second, we will use partitioning routines to split the job set into $M$ bags. We then learn the number of machines and need to schedule the bags on machines. In different cases, we will use different combinations of partitioning and scheduling routines. We then show that, for each case, the resulting schedule is $\frac{5}{3}(1+\epsilon)$ robust.

In Section 3.1, we will give our partitioning routines and prove some properties about each one. In Section 3.2, we will describe how to assemble the jobs into bags and then schedule them on machines.

\subsection{Partitioning}

In this subsection, we will describe three useful partitioning algorithms, which will be used as subroutines in the main algorithm. Each one will take one or four sets of jobs and partition them into multiple bags with special properties, achieving some balance between the sizes of the bags and controlling the placement of large jobs. In all routines, we will assume that we process the jobs in sorted size order, largest-to-smallest.

We begin with the first partitioning algorithm, Partition I, which partitions a job set into two bags such that neither of them is too big. This partitioning algorithm will be useful when we want to partition a job set "evenly." It implements the LPT algorithm, sorting the jobs in non-increasing order and then repeatedly placing the next job in the least loaded bag. It appears in Algorithm 1.

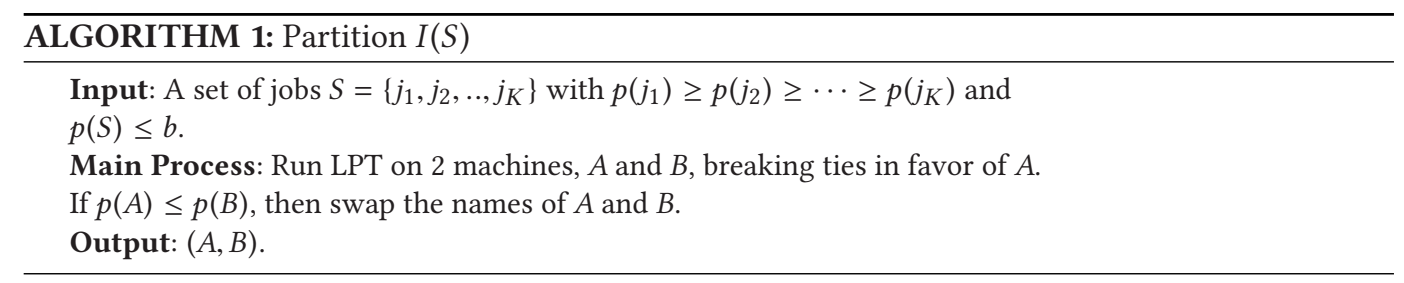

We now bound the sizes of the bags. Recall that the standard analysis of LPT shows that it is a $4 / 3-1 / 3 m=7 / 6$-approximation algorithm on two machines [8]. The bounds that we give are tight and are not implied by the standard analysis of LPT.

Lemma 3.1. Let $(A, B)$ be the output of Partition I, then $p(A)=\max \{p(A), p(B)\} \leq$ $\max \left\{p\left(j_{1}\right), 2 b / 3\right\}$. If the maximum is achieved by $p\left(j_{1}\right)$, then $A=\left\{j_{1}\right\}$.

Proof. If $j_{1}$ has larger processing time than all the other jobs combined, i.e., $p\left(j_{1}\right) \geq \sum_{i=2}^{K} p\left(j_{i}\right)$, then for $i=2, . ., K$, LPT will put $j_{i}$ in $B$ and $\max \{p(A), p(B)\}=p(A)=p\left(j_{1}\right)$.

Next consider the case when $p\left(j_{1}\right)<\sum_{i=2}^{K} p\left(j_{i}\right)$. We use $d_{t}$ to denote the difference in the loads of $A$ and $B$ after adding job $j_{t}$ (note that $d_{K}=p(A)-p(B)$ ). Pick $k$ as the smallest integer such that $p\left(j_{1}\right)<\sum_{i=2}^{k} p\left(j_{i}\right)$, clearly $k \geq 3$. Then, $p\left(j_{k}\right) \geq d_{k}=\sum_{i=2}^{k} p\left(j_{i}\right)-p\left(j_{1}\right)$, since $\sum_{i=2}^{k-1} p\left(j_{i}\right) \leq p\left(j_{1}\right)$. When we decide where to put $j_{i+1}$, the difference between the loads of $A$ and $B$ is $d_{i}$, and we put $j_{i+1}$ in the bag with less load. Hence, we must have $d_{i+1} \leq \max \left\{p\left(j_{i+1}\right), d_{i}\right\}$. Then inductively $d_{K} \leq \max \left\{p\left(j_{K}\right), p\left(j_{K-1}\right), . ., p\left(j_{k+1}\right), p\left(j_{k}\right), d_{k}\right\}$. Because the jobs are indexed largest to smallest, and because $k \geq 3$, we can simplify the previous inequality to $d_{K} \leq p\left(j_{3}\right) \leq b / 3$ (recall that $d_{k} \leq j_{k}$ ). Since $p(A)+p(B)=b$, it immediately follows that $\max \{p(A), p(B)\} \leq 2 b / 3$.

Since we almost always need to put multiple bags on one machine, we want to create enough small bags to control the makespan. This demand leads to the next two partitioning algorithms, in which we partition job sets to bags such that half of them are small enough and the others are 
not too big. The first one, Partition II, works for the case when we have a job set with at most one large job. We will place the job with the largest processing time in set $A$ and then fill set $B$ greedily up to $b / 3$ and then place the remaining jobs in $A$. The details appear in Algorithm 2.

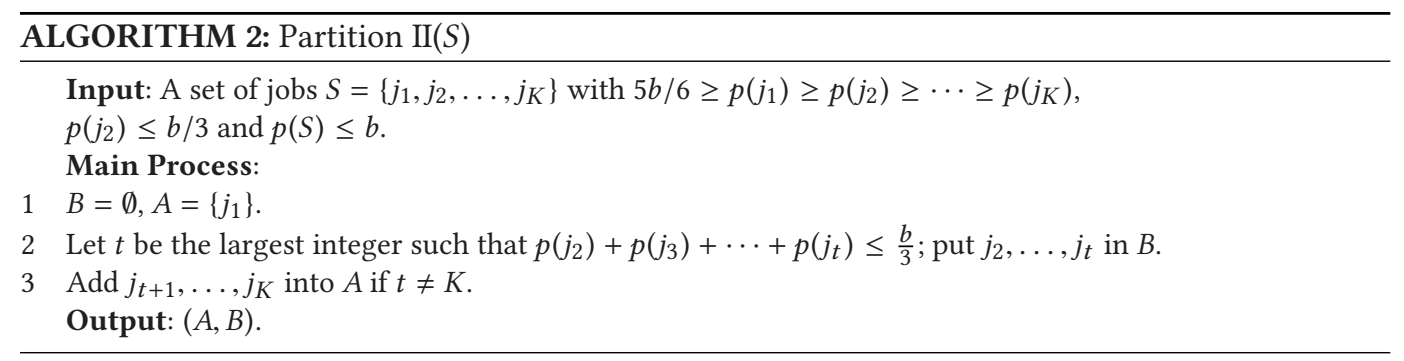

Lemma 3.2. Let $(A, B)$ be the output of Partition II, then $p(A) \leq 5 b / 6$ and $p(B) \leq b / 3$.

Proof. By line 2, clearly we have $p(B) \leq b / 3$. Suppose, for a contradiction, that $p(A)>5 b / 6$. Then $t \neq K$ and it follows that $p\left(j_{1}\right)+\sum_{i=t+1}^{K} p\left(j_{i}\right)>5 b / 6$. Since $\sum_{i=1}^{K} p\left(j_{i}\right) \leq b$, we must have $\sum_{i=2}^{t} p\left(j_{i}\right)=\sum_{i=1}^{K} p\left(j_{i}\right)-\left(p\left(j_{1}\right)+\sum_{i=t+1}^{K} p\left(j_{i}\right)\right)<b / 6$.

But, since $\sum_{i=2}^{t+1} p\left(j_{i}\right)>b / 3$, we also have $p\left(j_{t+1}\right)=\sum_{i=2}^{t+1} p\left(j_{i}\right)-\sum_{i=2}^{t} p\left(j_{i}\right)>b / 3-b / 6=b / 6$. Note that $t \geq 2$, hence $p\left(j_{2}\right) \geq p\left(j_{t+1}\right)>b / 6>\sum_{i=2}^{t} p\left(j_{i}\right)$, a contradiction.

The last partitioning algorithm, Partition III, will handle the case when we have at least two large jobs and Partition II is not working. Specifically, we will take four job sets as input; one of them, $S_{1}$, has two large $(\geq b / 3)$ jobs, another, $S_{2}$, has only small $(<b / 3)$ jobs, and the other two, $S_{3}$ and $S_{4}$, have no jobs greater than $2 b / 3$. We partition them into eight bags, such that we have four small bags and four bags that are not too big. The algorithm first puts the second biggest job from $S_{1}$ into $A_{1}$ and the remaining jobs from $S_{1}$ into $A_{2}$. It then greedily puts jobs from $S_{2}$ into $A_{1}$ and $A_{2}$ as long as they do not cause the load to go over $5 b / 6$. By greedily, here, we mean that it goes through the jobs in non-increasing order of processing time, and if the job can fit on $A_{1}$ or $A_{2}$, we place it on one that it fits. We then use Partition I on the remaining jobs of $S_{2}$, running LPT to place jobs on $B_{1}$ and $B_{2}$. We also use Partition I to partition $S_{3}$ to $\left(A_{3}, B_{3}\right)$, and $S_{4}$ to $\left(A_{4}, B_{4}\right)$. We will show that either $p\left(B_{1}\right)+p\left(B_{3}\right)$ and $p\left(B_{2}\right)+p\left(B_{4}\right)$ are both not too big or we can switch jobs to ensure that neither is. The details of the algorithm appear in Algorithm 3.

Lemma 3.3. Let $\left(A_{1}, A_{2}, A_{3}, A_{4}, B_{1}, B_{2}, B_{3}, B_{4}\right)$ be the output of Partition III. These bags satisfy:

(1) $p\left(A_{i}\right) \leq 5 b / 6$, for $i=1,2,3,4$;

(2) $p\left(B_{i}\right) \leq b / 2$, for $i=1,2,3,4$;

(3) $p\left(B_{1}\right)+p\left(B_{3}\right) \leq 5 b / 6, p\left(B_{2}\right)+p\left(B_{4}\right) \leq 5 b / 6$.

Proof. Let $k_{i j}$ denote the load of jobs from $S_{i}$ that are placed in bag $A_{j}$. Note that the jobs in bags $B_{1}$ and $B_{2}$ all come from $S_{2}$. Thus, $k_{11}+k_{12} \leq b$ and $k_{21}+k_{22}+p\left(B_{1}\right)+p\left(B_{2}\right) \leq b$. For $i=1,2$, define the non-negative slack in each $A_{i}$ as $\delta_{i}=5 b / 6-k_{1 i}-k_{2 i}$ and $\delta=\max \left\{\delta_{1}, \delta_{2}\right\}$.

Note that at line $1, p\left(A_{1}\right)=p\left(x_{2}\right) \leq b / 2$ and $p\left(A_{2}\right)=p\left(S_{1}\right)-p\left(x_{2}\right) \leq b-b / 3 \leq 2 b / 3$. Since we call Partition I to separate $S_{3}$ and $S_{4}$, by Lemma 3.1, $p\left(A_{3}\right) \leq 2 b / 3, p\left(A_{4}\right) \leq 2 b / 3$ at line 6 . Since for $i=1,2,3$, 4 , we will only add job to $A_{i}$ as long as the load does not exceed $5 b / 6$, condition 1 holds.

We now consider conditions 2 and 3. By line 2, any job in $B_{1} \cup B_{2}$ has load greater than $\delta$. First, consider the case when $B_{1} \cup B_{2}$ contains at most two job. Then, $p\left(B_{1}\right) \leq b / 3$ and $p\left(B_{2}\right) \leq b / 3$. Note that also $p\left(B_{3}\right) \leq p\left(S_{3}\right) / 2 \leq b / 2$ and similarly $p\left(B_{4}\right) \leq b / 2$, hence the claim follows. 


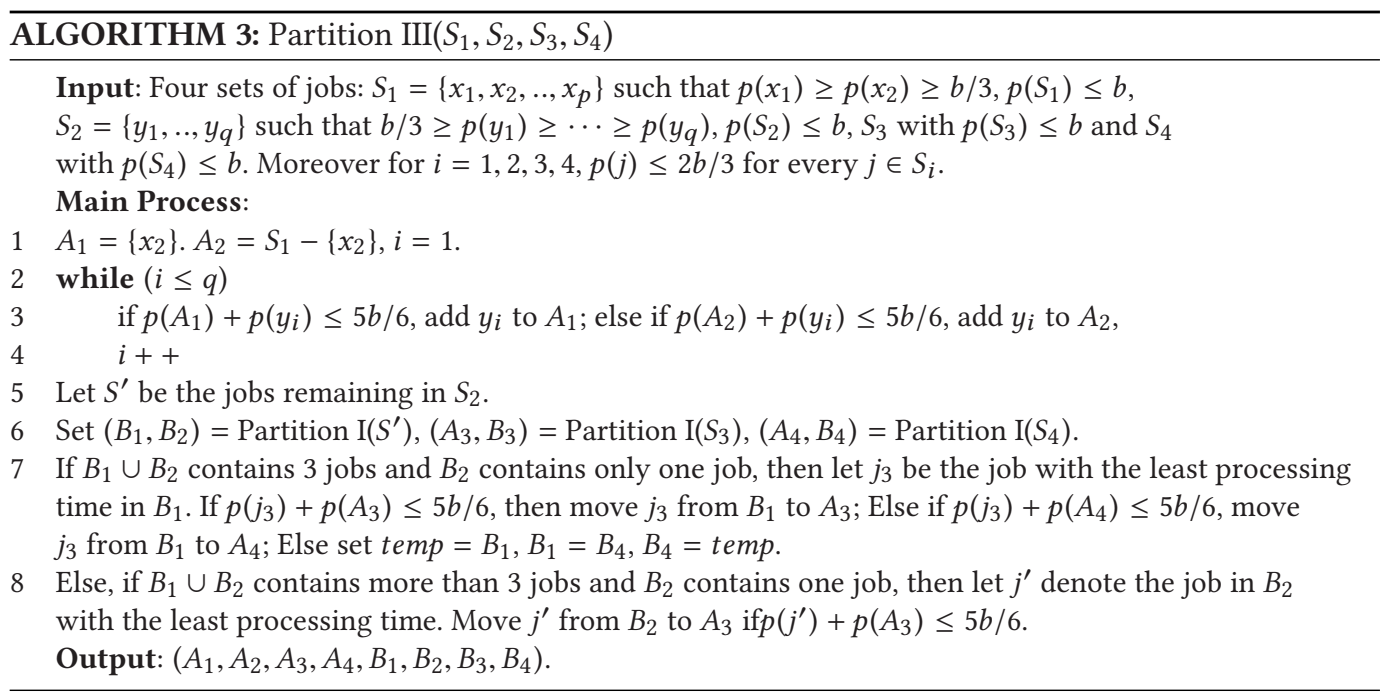

Next, we consider the case when $B_{1} \cup B_{2}$ contains $l \geq 3$ job before line 7 . Note that

$$
\begin{aligned}
& p\left(B_{1}\right)+p\left(B_{2}\right) \leq b-k_{21}-k_{22} \\
& =b-\left(\frac{5 b}{6}-\delta_{1}-k_{11}\right)-\left(\frac{5 b}{6}-\delta_{2}-k_{12}\right) \\
& \leq \frac{b}{3}+2 \delta .
\end{aligned}
$$

If both $B_{1}$ and $B_{2}$ contain at least 2 jobs, then $p\left(B_{1}\right) \geq 2 \delta$ and $p\left(B_{2}\right) \leq b / 3+2 \delta-p\left(B_{1}\right) \leq b / 3$. Similarly, $p\left(B_{2}\right) \leq b / 3$, hence the claim follows.

Next, we consider the case when $l=3$. If $B_{1}$ contains only one job, then $b / 3 \geq p\left(B_{1}\right) \geq p\left(B_{2}\right)$ and the claim follows. We may assume $B_{1}=\left\{j_{2}, j_{3}\right\}, B_{2}=\left\{j_{1}\right\}$ and $p\left(j_{1}\right) \geq p\left(j_{2}\right) \geq p\left(j_{3}\right)$. Since $k_{11}=p\left(x_{2}\right) \leq b / 2$ and $5 b / 6-k_{11} \geq b / 3 \geq p\left(y_{1}\right)$, we will always put $y_{1}$ in $A_{1}$. It implies that $p\left(y_{1}\right)+p\left(j_{1}\right)+p\left(j_{2}\right)+p\left(j_{3}\right) \leq p\left(S_{2}\right) \leq b$ jobs and $j_{3} \leq\left(p\left(y_{1}\right)+p\left(j_{1}\right)+p\left(j_{2}\right)+p\left(j_{3}\right)\right) / 4 \leq b / 4$. If in line 7 we move $j_{3}$ to either $A_{3}$ or $A_{4}$, then clearly claim follows, since after line 7 , both $B_{1}$ and $B_{2}$ contains one job and thus have load at most $b / 3$. So, we may assume that before line 7 , $p\left(A_{3}\right)>5 b / 6-p\left(j_{3}\right) \geq 5 b / 6-b / 4=7 b / 12$, and then $p\left(B_{3}\right) \leq b-p\left(A_{3}\right)<5 b / 12$. Similarly, we have $p\left(B_{4}\right)<5 b / 12$. Hence, $p\left(B_{3}\right)+p\left(B_{4}\right)<5 b / 6$ before line 7 . Note also that $p\left(j_{2}\right)+p\left(j_{3}\right) \leq$ $p\left(j_{1}\right)+p\left(y_{1}\right)$, hence $p\left(j_{2}\right)+p\left(j_{3}\right) \leq p\left(S_{2}\right) / 2 \leq b / 2$. So before line $7, p\left(B_{1}\right) \leq b / 2$ and $p\left(B_{2}\right) \leq b / 3$. Recall that we will swap the name of $B_{1}$ and $B_{4}$, hence the claim follows.

The last case is when $l \geq 4$ and one of $B_{1}$ and $B_{2}$ contains only one job. If $B_{1}$ contains only one job, then $b / 3 \geq p\left(B_{1}\right) \geq p\left(B_{2}\right)$ and the claim follows. We may assume that $B_{2}=\left\{j_{1}\right\}$ and $B_{1}=$ $\left\{j_{2}, j_{3}, \ldots, j_{l}\right\}$ with $p\left(j_{1}\right) \geq p\left(j_{2}\right) \geq \cdots \geq p\left(j_{l}\right)$. Then by Partition I, $p\left(j_{2}\right)+\cdots+p\left(j_{l-1}\right) \leq p\left(B_{1}\right) \leq$ $b / 3$. Recall that $l \geq 4$, hence $p\left(j_{l}\right) \leq\left(p\left(j_{2}\right)+\cdots+p\left(j_{l-1}\right)\right) /(l-2) \leq b / 6$. By Lemma 3.1, $p\left(A_{3}\right) \leq$ $2 b / 3$. Thus, $p\left(A_{3}\right)+p\left(j_{k}\right) \leq 5 b / 6$. So, in line 8 we will always move $j_{k}$ to $A_{3}$. After line $8, p\left(B_{2}\right)=$ $p\left(j_{2}\right)+\cdots+p\left(j_{l-1}\right) \leq b / 3$. Hence, the claim also follows.

\subsection{Packing and Scheduling}

In the previous subsection, we gave different algorithms to partition jobs into bags. We will now show how to use these algorithms in conjunction with additional algorithms to schedule the bags (and thus jobs) onto machines. Recall that opt ${ }_{M / 2}^{\prime}$ is the value of the makespan obtained by running 
the PTAS on a scheduling instance with $M / 2$ machines and $b=$ opt $_{M / 2}^{\prime}$. Two simple facts we will use throughout this section are that opt ${ }_{m}$ is non-increasing with $m$, the number of machine, and that $2 \mathrm{opt}_{M} \geq \mathrm{opt}_{M / 2}$, which implies that $\mathrm{opt}_{M} \geq \frac{\mathrm{opt}_{M / 2}^{\prime}}{2(1+\epsilon)}=\frac{b}{2(1+\epsilon)}$.

We now prove our bound of $\frac{5}{3}+\epsilon$ by considering three different cases. The first case is when $o p t_{M}^{\prime} \geq 3 b / 5$, which implies that the PTAS of the jobs on $M / 2$ is good enough for $M$ machines. We remark that if this case does not hold then there is no job with processing time greater than $3 b / 5$. The next case is when $o p t_{3 M / 4}^{\prime} \leq 4 b / 5$, which implies that we can start with the PTAS of the jobs on $3 M / 4$ machines and do not need to split all job sets. The last and the hardest case is when $o p t_{3 M / 4}^{\prime}>4 b / 5$ and $o p t_{M}^{\prime}<3 b / 5$. In this case, we will start with the PTAS on $M / 2$ and split each job sets according to its structure. Before going to the detail of those three cases, we start with a lemma that gives a sufficient condition for bags to give a $\frac{5}{3}(1+\epsilon)$ robust ratio when the number of machine is less than $M / 2$. It will be used in both the second and the last case.

LEMMA 3.4. Let $\left\{S_{1}, \ldots, S_{M}\right\}$ denotes $M$ bags such that for $i=1,2, \ldots, M / 2, p\left(S_{i}\right) \leq 5 b / 6$, and for $i=M / 2+1, M / 2+2, \ldots, M, p\left(S_{i}\right) \leq 2 b / 3$. Then, the following algorithm yields a schedule for the bags with a makespan of at most $5(1+\epsilon) \operatorname{opt}_{m} / 3$ for any $m \in[1, M / 2)$.

(1) If $M / 4 \leq m<M / 2$, then for $i=1,2, \ldots, m$, put $S_{i}$ on machine $\mathrm{i}$; for $i=m+1, m+$ $2, \ldots, M / 2$, put $S_{i}$ on machine $i-m$; for $i=M / 2+1, \ldots, M$, put $S_{i}$ on the machine with the least load.

(2) If $\frac{M}{2(k+1)} \leq m<\frac{M}{2 k}$ for some integer $k \geq 2$, then sort $\left\{S_{1}, S_{2}, \ldots, S_{M}\right\}$ in decreasing order according to their processing times. For $i=1, \ldots, m$, put $S_{i}$ on machine $i$; for $i=m+$ $1, \ldots, 2 m$, put $S_{i}$ on machine $i-m$; for $i=2 m+1, \ldots, M$, put $S_{i}$ on the machine with the least load.

Proof. First note that since $m<M / 2$, opt ${ }_{m} \geq$ opt $_{M / 2} \geq b /(1+\epsilon)$. Let machine $r$ be the machine with the largest load and among the bags scheduled on machine $r, S_{t}$ is the one with the largest index. For the case $M / 4 \leq m<M / 2$, if $t>M / 2$, then we know $p\left(S_{t}\right) \leq 2 b / 3 \leq 2(1+$ $\epsilon)$ opt $_{m} / 3$ and the makespan of our algorithm is at most $p\left(S_{t}\right)+$ opt $_{m} \leq 5(1+\epsilon)$ opt $_{m} / 3$. Else $t \leq M / 2$, then we know that we only put two bags on machine $r$, and therefore the makespan is at most $2 \cdot 5 b / 6=5 b / 3 \leq 5(1+\epsilon)$ opt $_{m} / 3$.

For the case $\frac{M}{2(k+1)} \leq m<\frac{M}{2 k}$ with $k \geq 2$, if $t \leq 2 m$, then similarly we know that we only put two bags on machine $r$, and therefore the makespan is at most $2 \cdot 5 b / 6=5 b / 3 \leq 5(1+\epsilon) \operatorname{opt}_{m} / 3$. So, we may assume that $t \geq 2 m+1$. Since $\sum_{i=1}^{2 m} p\left(S_{i}\right) \geq \sum_{i=1}^{2 m} p\left(S_{t}\right)=2 m p\left(S_{t}\right)$, opt ${ }_{m} \geq \sum_{i=1}^{2 m} p\left(S_{i}\right) / m \geq$ $2 p\left(S_{t}\right)$. This implies that $p\left(S_{t}\right) \leq \mathrm{opt}_{m} / 2$ and the makespan of our algorithm is at most $p\left(S_{t}\right)+$ $\mathrm{opt}_{m} \leq 3 \mathrm{opt}_{m} / 2$.

3.2.1 Case I: $\mathrm{opt}_{M}^{\prime} \geq 3 b / 5$. The first case is quite simple. We start with $\operatorname{PTAS}(M / 2)$, and partition each job set into two bags using Partition I. Those are our bags. For scheduling, if $m \geq M / 2$, then we just revert to the $M / 2$ machine schedule, leaving the remaining machines empty. If $m<M / 2$, then we can run LPT to the bags on the machines. The details appear in Algorithm 4 .

Lemma 3.5. If opt $_{M}^{\prime} \geq 3 b / 5$, then Algorithm 4 is $\frac{5}{3}(1+\epsilon)$-robust.

Proof. For $m \geq M / 2$,

$$
\frac{A L G(m, M)}{\mathrm{opt}_{m}} \leq \frac{\mathrm{opt}_{M / 2}^{\prime}}{\mathrm{opt}_{M}} \leq \frac{b}{\frac{\mathrm{opt}_{M}^{\prime}}{1+\epsilon}} \leq \frac{b}{\frac{3 b / 5}{1+\epsilon}}=(1+\epsilon) \frac{5}{3} .
$$

For $m<M / 2$, opt ${ }_{m} \geq$ opt $_{M / 2} \geq b /(1+\epsilon)$. We may assume the bags are $S_{1}^{\prime}, \ldots, S_{M}^{\prime}$ such that $p\left(S_{1}^{\prime}\right) \geq p\left(S_{2}^{\prime}\right) \cdots \geq p\left(S_{M}^{\prime}\right)$. Let $k$ be the number such that for $i \leq k, p\left(S_{i}^{\prime}\right)>2 b / 3$ and for $i>k$, 


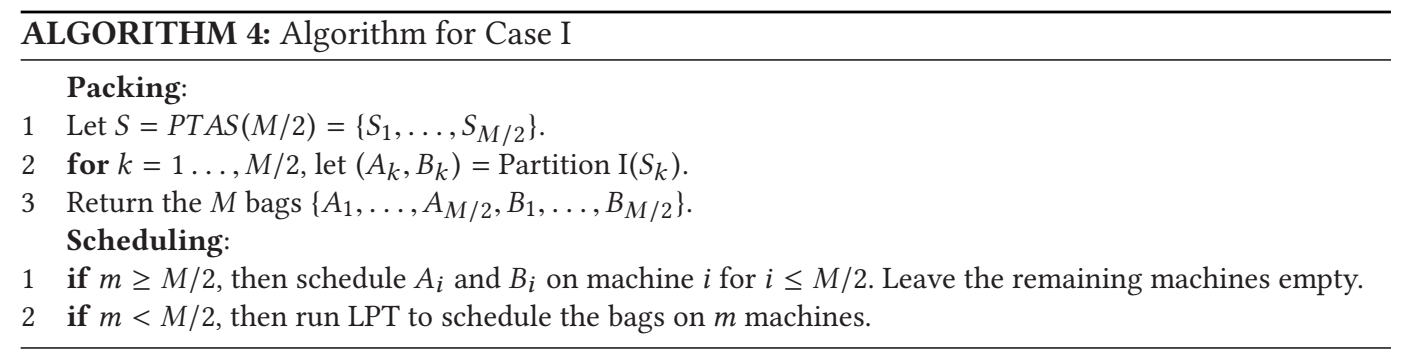

$p\left(S_{i}^{\prime}\right) \leq 2 b / 3$. Recall that each bag $S_{i}^{\prime}$ comes from running Partition I on some job set $S_{j}$ with $p\left(S_{j}\right) \leq b$. Hence, by Lemma 3.1, either $p\left(S_{i}^{\prime}\right) \leq 2 b / 3$ or it is a bag with a single job. Specifically, $S_{1}^{\prime}, \ldots, S_{k}^{\prime}$ all contain only one job. Let the jobs be $j_{1}, j_{2}, \ldots, j_{k}$, respectively. Let machine $r$ be the machine with the largest load and among the bags scheduled on machine $r, S_{t}$ is the one with the largest index. If $t>k$, then we know $p\left(S_{t}\right) \leq 2 b / 3 \leq 2(1+\epsilon)$ opt $_{m} / 3$. By the property of LPT, the makespan of our algorithm is at most $p\left(S_{t}\right)+\mathrm{opt}_{m} \leq 5(1+\epsilon) \mathrm{opt}_{m} / 3$. If $t \leq k$, then the makespan of our algorithm is equal to the makespan of running LPT on jobs $\left\{j_{1}, j_{2}, \ldots, j_{k}\right\}$, which is at most $4 / 3$ times the minimum makespan of scheduling $\left\{j_{1}, j_{2}, \ldots, j_{k}\right\}$ on $m$ machines [8]. Since the minimum makespan of scheduling $\left\{j_{1}, j_{2}, \ldots, j_{k}\right\}$ on $m$ machines is at most opt ${ }_{m}$, our makespan in this case is at most $4 \mathrm{opt}_{m} / 3$.

3.2.2 Case II: opt $_{M}^{\prime}<\frac{3 b}{5}$ and opt $_{3 M / 4}^{\prime} \leq 4 b / 5$. In this case, we will use the PTAS schedule for $3 M / 4$ machines as input to Partition I. For $S_{i} \in \operatorname{PTAS}(3 M / 4)$, we call $S_{i}$ bad if $S_{i}$ contains a job with processing time at least $\frac{2}{3} \mathrm{opt}_{3 M / 4}^{\prime}$. For the packing step, we will run Partition I on $M / 4$ job sets, try to avoid a bad set if possible, and then schedule the bags on machines. For the scheduling step, we will have several cases, based on how many bad machines that we have. The details appear in Algorithm 5.

\section{ALGORITHM 5: Algorithm for Case II}

\section{Packing:}

1 Let $S=P T A S(3 M / 4)=\left\{S_{1}, \ldots, S_{3 M / 4}\right\}$. Let $v$ be the number of bad sets in $S$. Rename the sets so that $S_{1}, \ldots, S_{v}$ are bad sets.

2 For $M / 2<k \leq 3 M / 4$, let $\left(A_{k}, B_{k}\right)=$ Partition $\mathrm{I}\left(S_{k}\right)$.

3 Return the $M$ bags $\left\{S_{1}, S_{2}, \ldots, S_{M / 2}, A_{M / 2+1}, \ldots, A_{3 M / 4}, B_{M / 2+1}, . ., B_{3 M / 4}\right\}$.

Scheduling:

1 if $m \geq 3 M / 4$, then schedule $S_{i}$ on machine $i$ for $i \leq M / 2$, and schedule $A_{j}, B_{j}$ on machine $j$ for $j>M / 2$. Leave the remaining machines empty.

2 if $v>M / 2$ and $v \leq m<3 M / 4$, then first put $S_{1}, S_{2}, \ldots, S_{M / 2}, A_{M / 2+1} \cup B_{M / 2+1}, \ldots, A_{v} \cup B_{v}$ on $v$ machines, respectively, then schedule the rest of the bags with at most one in each machine.

3 if $v>M / 2$ and $M / 2 \leq m<v-1$, or $v \leq M / 2$ and $M / 2 \leq m<3 M / 4$, then schedule the bags arbitrarily such that each machine contains at most two bags and at most one bag comes from $\left\{S_{1}, \ldots, S_{M / 2}\right\}$.

4 if $m<M / 2$, then we follow the strategy in Lemma 3.4.

LEMMA 3.6. If $\mathrm{opt}_{M}^{\prime}<\frac{3 b}{5}$ and $\mathrm{opt}_{3 M / 4}^{\prime} \leq 4 b / 5$, then Algorithm 5 is $\frac{5}{3}(1+\epsilon)$-robust.

Proof. For $m \leq 3 M / 4$, the load on each machine is at most opt $t_{3 M / 4}^{\prime} \leq 4 b / 5$; hence, we have

$$
\frac{A L G(m, M)}{\mathrm{opt}_{m}} \leq \frac{\mathrm{opt}_{3 M / 4}^{\prime}}{\mathrm{opt}_{M}} \leq \frac{4 b / 5}{\frac{b}{2(1+\epsilon)}}<(1+\epsilon) \frac{5}{3} .
$$


By Lemma 3.1, for $k>\max \{v, M / 2\}, p\left(B_{k}\right) \leq p\left(A_{k}\right) \leq \frac{2}{3} \mathrm{opt}_{3 M / 4}^{\prime}$. If $v>M / 2$ and $v \leq m<$ $3 M / 4$ or $v \leq M / 2$ and $M / 2 \leq m<3 M / 4$, then the load on each machine is at most $\mathrm{opt}_{3 M / 4}^{\prime}+$ $\max _{i>v}\left\{p\left(A_{i}\right)\right\} \leq \frac{5}{3} \mathrm{opt}_{3 M / 4}^{\prime}$, so we have

$$
\frac{A L G(m, M)}{\mathrm{opt}_{m}} \leq \frac{\frac{5}{3} \mathrm{opt}_{3 M / 4}^{\prime}}{\mathrm{opt}_{3 M / 4}} \leq(1+\epsilon) \frac{5}{3} .
$$

If $v>M / 2$ and $M / 2 \leq m<v-1$, then since the number of jobs with processing time at least $\frac{2}{3} \operatorname{opt}_{3 M / 4}^{\prime}$ is at least $v$, opt $v_{v-1} \geq \frac{4}{3} \operatorname{opt}_{3 M / 4}^{\prime}$. However, each machine has load at most $2 \mathrm{opt}_{3 M / 4}^{\prime}$. So we obtain

$$
\frac{A L G(m, M)}{\mathrm{opt}_{m}} \leq \frac{2 \mathrm{opt}_{3 M / 4}^{\prime}}{\mathrm{opt}_{v-1}} \leq \frac{2 \mathrm{opt}_{3 M / 4}^{\prime}}{\frac{4}{3} \mathrm{opt}_{3 M / 4}^{\prime}}<(1+\epsilon) \frac{5}{3} .
$$

Since opt ${ }_{M}^{\prime}<3 b / 5$, every job has processing time at most $3 b / 5$. Notice that for $i \leq M / 2$ and $j>M / 2, p\left(S_{i}\right) \leq \operatorname{opt}_{3 M / 4}^{\prime} \leq 4 b / 5$ and $p\left(B_{j}\right) \leq p\left(A_{j}\right) \leq \max \left\{3 b / 5,2 \operatorname{opt}_{3 M / 4}^{\prime} / 3\right\}=3 b / 5<2 b / 3$, we can apply Lemma 3.4 on the case $m<M / 2$.

3.2.3 Case III: $\mathrm{opt}_{M}^{\prime}<\frac{3 b}{5}$ and $\mathrm{opt}_{3 M / 4}^{\prime}>4 b / 5$. This final case is the most complicated one. We say a job is big if it has processing time at least $b / 3$. Let $S$ be a set of jobs with sum of the processing time at most $b$, we call the set 2-big if it has at least two big jobs. We call the set 1-big if it is not 2-big and has one big job. We call a set that is neither 1-big nor 2-big, 0-big. Give a set $S$ of job sets, we let $v_{i}(S)$ denote the number of $i$-big sets, and we assume that we have functions $i$-big $(S)$, which return an i-big set from $S$, assuming one exists. For a job set $S_{i}$, we use $S_{i}(j)$ to denote the $j$ th largest job in set $S_{i}$.

The main idea for packing here is to use the optimal schedule for $M / 2$ machines, but to split the jobs assigned to each machine into 2 bags. For each $S_{i}$, we want to partition it into two sets such that one is small enough and the other is not too big. We can use Partition II to achieve this goal, if and only if $S_{i}$ contains at most one big job, that is, $S_{i}$ is 0-big or 1-big. If $S_{i}$ is 2-big and thus contain two big jobs, then we try to group three 2-big job sets with one 0-big job set, and then partition them into eight sets such that four are small enough and four not too big, using Partition III. Roughly speaking, if many $S_{i}$ contain two big jobs, then we can give a good lower bound on opt, else we will have a good partition. The details appear in Algorithm 6.

LemMA 3.7. If $\mathrm{opt}_{M}^{\prime}<3 b / 5$ and $\mathrm{opt}_{3 M / 4}^{\prime}>4 b / 5$, then Algorithm 6 is $\frac{5}{3}(1+\epsilon)$-robust.

Proof. Since opt ${ }_{M}<3 b / 5$, every job has processing time at most $3 b / 5$. For $k=1,2,3, \ldots, 4 u$, by Lemma 3.2, we have $p\left(A_{k}\right) \leq 5 b / 6$ and $p\left(B_{k}\right) \leq b / 2$ and if moreover $k=4 t+1$ or $4 t+2$ for an integer $t, p\left(B_{k}\right)+p\left(B_{k+2}\right) \leq 5 b / 6$, and we call such $B_{k}$ and $B_{k+2}$ paired bags. For $k=4 u+1,4 u+$ $2, \ldots, 4 u+w$, we partition a 2-big job set $S_{i}$ into $\left\{A_{k}, B_{k}\right\}$ by putting the second biggest job in $B_{k}$ and the rest in $A_{k}$. Hence, $b / 3 \leq p\left(B_{k}\right) \leq b / 2, p\left(A_{k}\right)=p\left(S_{i}\right)-p\left(B_{k}\right) \leq b-b / 3=2 b / 3$. For $k=$ $4 u+w+1,4 u+w+2, . ., \frac{M}{2}$, by Lemma 3.3, we have $p\left(A_{k}\right) \leq 5 b / 6, p\left(B_{k}\right) \leq b / 3$ (note that after line 3 there is no 2-big job set left, and hence we can use Partition II in line 4).

Next consider the scheduling process in Algorithm 4. First consider the case $m \geq \max$ $\{3 M / 4, M / 2+w+2 u\}$. For $i \leq M / 2$, the load of each machine is at $\operatorname{most} \max _{i}\left\{p\left(A_{i}\right)\right\} \leq 5 b / 6$. For $i=M / 2+1, M / 2+2, \ldots, M / 2+2 u$, we always schedule a paired bags on the machines, so the load is still at most $5 b / 6$. For the rest of the machines, we schedule $B_{4 u+1}, \ldots, B_{M / 2}$ on them such that there are at most two bags on each machine, and if there are two bags, at most one of them is 


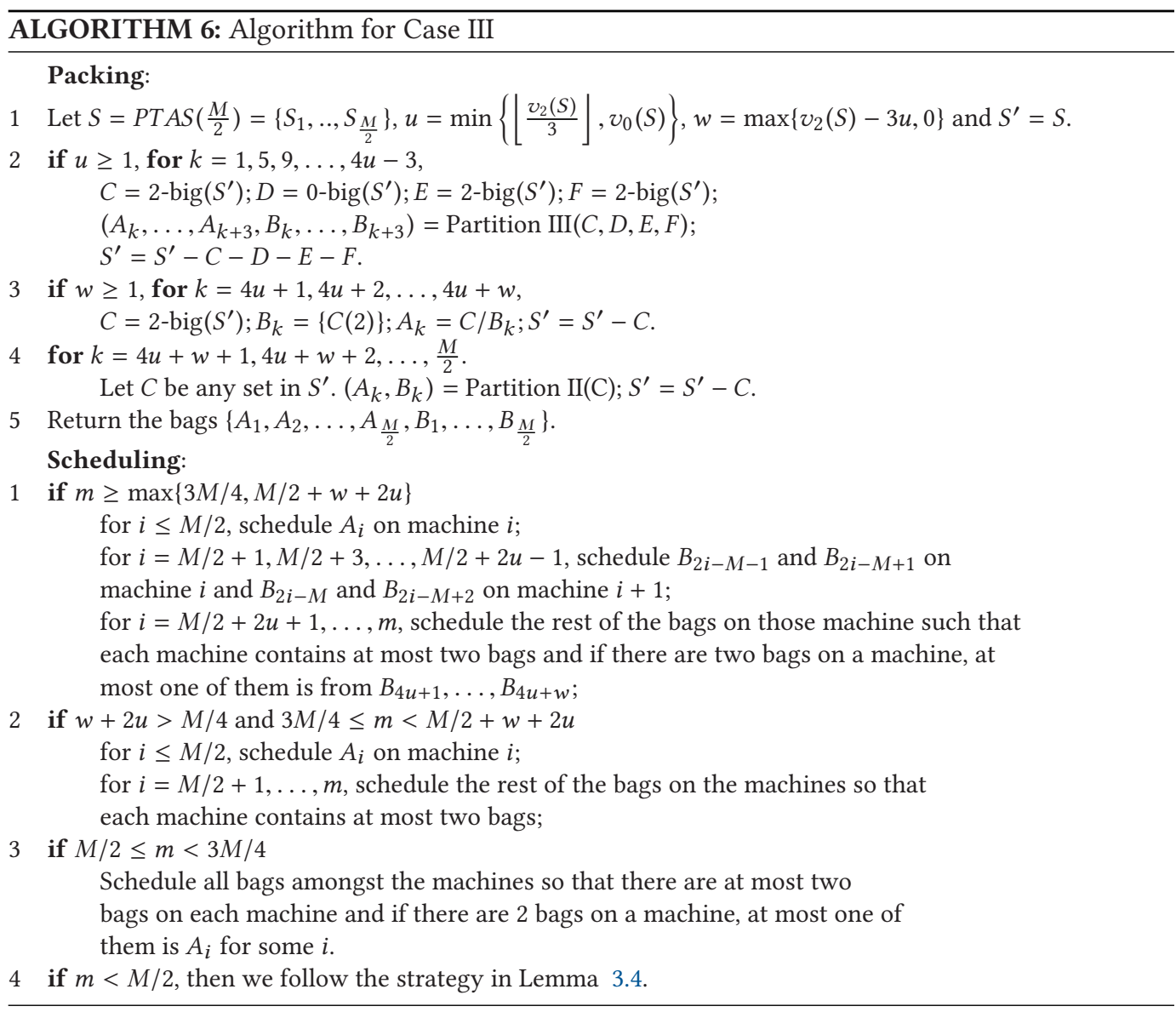

from $B_{4 u+1}, \ldots, B_{4 u+w}$; hence, the load is at most $b / 3+b / 2=5 b / 6$. Therefore,

$$
\frac{A L G(m, M)}{\mathrm{opt}_{m}} \leq \frac{5 b / 6}{\mathrm{opt}_{M}} \leq \frac{5 b / 6}{\frac{b}{2(1+\epsilon)}}=(1+\epsilon) \frac{5}{3} .
$$

Next, consider the case $w+2 u>M / 4$ and $3 M / 4 \leq m<M / 2+w+2 u$. Since $4 u \leq v_{2}(S)+$ $v_{0}(S) \leq M / 2,2 u \leq M / 4$ and $v_{2}(S)-3 u=w \geq 1$. If $u=\left\lfloor v_{2}(S) / 3\right\rfloor<v_{0}(S)$, since $v_{2}(S) \geq 3 u+$ 1 , then $v_{2}(S)=3 u+1$ or $3 u+2$. If $v_{2}(S)=3 u+1$, then $M / 4<w+2 u=v_{2}(S)-u=2 u+1$ and $M / 4 \leq 2 u$, but $M / 2 \geq v_{2}(S)+v_{0}(S)>3 u+1+u$, a contradiction. If $v_{2}(S)=3 u+2$, then $M / 4<v_{2}(S)-u=2 u+2$ and $M / 4 \leq 2 u+1$, but $M / 2 \geq v_{2}(S)+v_{0}(S)>3 u+2+u$, a contradiction. So, we have $u \geq v_{0}(S)$ and $w+2 u=v_{2}(S)-u \leq v_{2}(S)-v_{0}(S)$. Note that in total we have at least $2 v_{2}(S)+v_{1}(S)=M / 2+v_{2}(S)-v_{0}(S)$ jobs with processing time at least $b / 3$. Hence, $\operatorname{opt}_{M / 2+w+2 u-1} \geq 2 b / 3$. However, in this case the load on each machine is at most $\max \left\{\max _{i}\left\{A_{i}\right\}, 2 \max _{i}\left\{B_{i}\right\}\right\} \leq b$ :

$$
\frac{A L G(m, M)}{\mathrm{opt}_{m}} \leq \frac{b}{\mathrm{opt}_{M / 2+w+2 u-1}} \leq \frac{b}{2 b / 3}<(1+\epsilon) \frac{5}{3} .
$$


In the case $M / 2 \leq m<3 M / 4$, the load on each machine is at $\operatorname{most}^{\max _{i}}\left\{A_{i}\right\}+\max _{i}\left\{B_{i}\right\} \leq$ $5 b / 6+b / 2=4 b / 3$. Therefore, we have

$$
\frac{A L G(m, M)}{\mathrm{opt}_{m}} \leq \frac{4 b / 3}{\mathrm{opt}_{3 M / 4}} \leq(1+\epsilon) \frac{4 b / 3}{4 b / 5}=(1+\epsilon) \frac{5}{3} .
$$

For the case when $m<M / 2$, by Lemma 3.4 the claim follows.

By combining Lemmas 3.5, 3.6, and 3.7, we can deduce the following lemma. The running time comes from calling the PTAS [10] three times, various sorting and heap data structure operations needed to implement LPT and the other algorithms. The $M$ term is in the running time because of the need to perform operations on the $M$ bags.

Lemma 3.8. When $M$ is divisible by 4, Algorithms 4, 5, and 6 together give a $\frac{5}{3}(1+\epsilon)$-robust algorithm with running time $2^{\tilde{O}(1 / \epsilon)}+O((M+n) \log n)$.

Recall that the algorithm uses the terms $M / 2$ and $3 M / 4$ and assumes that they are integers. When $M$ is not divisible by 4 , the algorithm and analysis still work with some small changes.

Lemma 3.9. When $M$ is not divisible by 4 , there exists an algorithm gives a $\frac{5}{3}(1+\epsilon)$-robust algorithm with running time $2^{\tilde{O}(1 / \epsilon)}+O((M+n) \log n)$.

Proof. We prove this Lemma by making necessary modifications on Algorithms 4, 5, and 6 depending on the remainder of $M$ divided by 4 . We first consider the case when $M=4 r+2$, for some nonnegative integer $r$. Then, we can still follow Algorithm 4. For Algorithm 6, we replace every $M / 4$ by $r+1$ and every $3 M / 4$ by $3 r+2$. And then Lemma 3.7 still holds with the same argument under the assumption that $o p t_{3 r+2}^{\prime}>4 b / 5$ instead of $o p t_{3 M / 4}^{\prime}>4 b / 5$. So the only remaining case is $o p t_{3 r+2}^{\prime} \leq 4 b / 5$, and we can follow Algorithm 5 with small modifications. In the packing phase of Algorithm 5, we will only partition $r$ sets and return with $M=4 r+2$ bags $\left\{S_{1}, \ldots, S_{2 r+2}, A_{2 r+3}, \ldots, A_{3 r+2}, B_{2 r+3}, \ldots, B_{3 r+2}\right\}$. Then, in the scheduling phase, line 1 holds for $m \geq 3 r+2$, line 2 holds for $v>2 r+2$ and $v \leq m<3 r+2$, and line 3 holds for $v>2 r+2$ and $2 r+2 \leq m<v-1$, or $v \leq 2 r+2$ and $2 r+2 \leq m<3 r+2$. The corresponding part of Lemma 3.6 holds accordingly. The last case is when $m \leq 2 r+1$, and we can follow Lemma 3.4 with the same strategy except for replacing $M / 2$ with $2 r+2$ and $M / 4$ with $r+1$.

Next, we consider the case when $M=4 r+1$, for some nonnegative integer $r$. In this case, we denote $b=o p t_{2 r+1}^{\prime}$ instead of $o p t_{M / 2}^{\prime}$, and we replace $M / 2$ by $2 r+1$ in all three algorithm. Then, we still have that $o p t_{M} \geq \frac{b}{1+\epsilon}$. For Algorithm 4, in the packing phase, we start with $S=P T A S(2 r+$ 1) in line 1 , and we run partition I for $S_{1}, S_{2} \ldots, S_{2 r}$ in line 2 . Then, we can still return $M$ bags $\left\{A_{1}, \ldots, A_{2 r}, B_{1}, \ldots, B_{2 r}, S_{2 r+1}\right\}$ in line 3. We may assume that either $S_{2 r+1}$ contains a job with processing time greater than $2 b / 3$ or every job has processing time at most $2 b / 3$. For the case $m \geq 2 r+1$, we follow line 1 of the scheduling phase of Algorithm 4 and clearly the robust ratio still holds. Next, we consider the case for $m \leq 2 r$, and we run LPT to schedule the bags on $m$ machines. Recall that for $i=1,2, \ldots, 2 r$, either $p\left(A_{i}\right) \leq 2 b / 3$ or $A_{i}$ is a bag with a single job, and same for $B_{i}$. To prove the ratio still hold, it is sufficient to show that the bags finish last has size at most $2 b / 3$. If $S_{2 r+1}$ does not contain a job with processing time greater than $2 b / 3$, then every job has processing time at most $2 b / 3$. We may assume that we run LPT with $S_{2 r+1}$ first (otherwise $p\left(S_{2 r+1}\right) \leq 2 b / 3$ ) and that $S_{2 r+1}$ is not the bag finishes last, since otherwise the makespan is at most $b$. But now the bags finishes last has size at most $2 b / 3$ and the claim holds. So, we may assume $S_{2 r+1}$ contains a job with processing time greater than $2 b / 3$, say $j$. Then the proof of Lemma 3.5 works except for the case when the machine finishes last contains only bags with single jobs and $S_{2 r+1}$. But its makespan is at most $4 o p t_{m} / 3+p\left(S_{2 r+1} \backslash\{j\}\right) \leq 5 o p t_{m} / 3$. For Algorithm 6, recall that we do it under the case 
when $o p t_{4 r+1}<3 b / 5<2 b / 3$, then it implies that for the sets in $\left\{S_{1}, S_{2}, \ldots, S_{2 r+1}\right\}=\operatorname{PTAS}(2 r+1)$, at most $2 r$ of which contain at least 2 big jobs. As we can only provide $4 r+1 \mathrm{bag}$, in the packing phase, we will return with the bags $\left\{A_{1}, \ldots, A_{2 r+1}, A^{\prime}=B_{1} \cup B_{3}, B_{2}, B_{4}, B_{5}, \ldots, B_{2 r+1}\right\}$ if $u \geq 1$ and $\left\{A_{1}, \ldots, A_{2 r+1}, A^{\prime}=B_{1} \cup B_{2 r+1}, B_{2}, B_{3}, \ldots, B_{2 r}\right\}$ otherwise. Then, $p\left(A^{\prime}\right) \leq 5 b / 6$, and we will treat it like an $A$-bag. Then in the scheduling phase, line 1 holds for $m \geq \max \{3 r+2,2 r+1+w+2 u\}$, line 2 holds for $w+2 u>r+1$ and $3 r+2 \leq m<2 r+1+w+2 u$, and line 3 holds for $2 r+2 \leq$ $m \leq 3 r+1$. The corresponding part of Lemma 3.7 holds accordingly under the assumption that $o p t_{3 r+1}>4 b / 5$. Also for the case when $m \leq 2 r+1$, we can follow Lemma 3.4 with the same strategy except for replacing $M / 2$ with $2 r+2$ and $M / 4$ with $r+1$. It left the case when $o p t_{3 r+1} \leq 4 b / 5$, and we follow Algorithm 5 with modifications. In the packing phase of Algorithm 5 , we will only partition $r$ sets and return with $M=4 r+1$ bags $\left\{S_{1}, \ldots, S_{2 r+1}, A_{2 r+2}, \ldots, A_{3 r+1}, B_{2 r+2}, \ldots, B_{3 r+1}\right\}$. Then in the scheduling phase, line 1 holds for $m \geq 3 r+1$, line 2 holds for $v>2 r+1$ and $v \leq m<$ $3 r+1$, and line 3 holds for $v>2 r+1$ and $2 r+1 \leq m<v-1$, or $v \leq 2 r+1$ and $2 r+1 \leq m<$ $3 r+1$. The corresponding part of Lemma 3.6 holds accordingly. Also for the case when $m \leq 2 r+1$, we can follow Lemma 3.4 similarly.

For the last case when $M=4 r+3$, for some nonnegative integer $r$. In this case, we denote $b=$ $o p t_{2 r+2}^{\prime}$ instead of $o p t_{M / 2}$, and we replace $M / 2$ by $2 r+2$ in all three algorithm. The modification of Algorithm 4 is exactly the same as in the case when $M=4 r+1$. For Algorithm 6, similarly, we have at most $2 r+1$ sets in $\operatorname{PTAS}(2 r+2)$, which contain at least 2 big jobs. in the packing phase, we will return with the bags $\left\{A_{1}, \ldots, A_{2 r+2}, A^{\prime}=B_{1} \cup B_{3}, B_{2}, B_{4}, B_{5}, \ldots, B_{2 r+2}\right\}$ if $u \geq 1$ and $\left\{A_{1}, \ldots, A_{2 r+2}, A^{\prime}=B_{1} \cup B_{2 r+2}, B_{2}, B_{3}, \ldots, B_{2 r+1}\right\}$ otherwise. Then in the scheduling phase, line 1 holds for $m \geq \max \{3 r+3,2 r+2+w+2 u\}$, line 2 holds for $w+2 u>r+1$ and $3 r+3 \leq m<2 r+$ $2+w+2 u$, and line 3 holds for $2 r+3 \leq m \leq 3 r+2$. The corresponding part of Lemma 3.7 holds accordingly. Also for the case when $m \leq 2 r+2$, we can follow Lemma 3.4 with the same strategy except for replacing $M / 2$ with $2 r+3$ and $M / 4$ with $r+2$. It left the case when $o p t_{3 r+2} \leq 4 b / 5$, and we follow Algorithm 5 with similar modifications as in the previous case. This finishes the proof of 3.9 .

In sum, we have the following main theorem.

THEOREM 3.10. There exists a $\left(\frac{5}{3}+\epsilon\right)$-robust algorithm with running time $2^{\tilde{O}(1 / \epsilon)}+O((M+$ n) $\log n)$.

\section{CONCLUSION AND OPEN PROBLEMS}

We initiate the idea of scheduling with uncertainty in a number of machines and give several results. In this article, we focus on the case when $m \in\{1,2,3, \ldots, M\}$, but there still exists a gap between the lower bound of $4 / 3$ and the upper bound of 5/3. It would be very interesting to close the gap. We can also generalize the idea to consider the case when $m \in[\alpha M, \beta M]$ to see whether we can give a better upper bound if we know a restriction on the possible number of machines in advance. Some of our partitioning lemmas in Section 3.1 can be generalized and may be useful in such analysis. For example, if we have a set $S=\left\{j_{1}, j_{2}, ., j_{K}\right\}$, with jobs satisfying $1-\alpha b / 2 \geq$ $p\left(j_{1}\right) \geq p\left(j_{2}\right) \geq \cdots \geq p\left(j_{K}\right), p\left(j_{2}\right) \leq \alpha b$, and $p(S) \leq b$, then we can use the idea of Partition II to partition $S$ to one set with load at most $1-\alpha b / 2$ and another set with load at most $\alpha b$. Partition III can also be generalized similarly.

It would be natural to consider other scheduling problems and other objectives, such as average completion time or flow time. Makespan is really a partitioning problem, but other objectives raise additional questions regarding the ordering of the jobs, which would be interesting to study. Finally, it might be interesting to consider the case where the number of machines come from a distribution and you need to schedule to minimize the expected makespan. 


\section{ACKNOWLEDGMENTS}

We are thankful to Tsvi Kopelowitz for many helpful discussions. We thank the anonymous reviewer who pointed out the simple 2-robust algorithm mentioned in Section 1.

\section{REFERENCES}

[1] S. Albers. 2013. Recent advances for a classical scheduling problem. In Proceedings of the Automata, Languages, and Programming. 4-14.

[2] S. Albers and M. Hellwig. 2014. Online makespan minimization with parallel schedules. In Proceedings of the Scandinavian Symposium and Workshops on Algorithm Theory. 13-25.

[3] S. Albers and G. Schmidt. 2001. Scheduling with unexpected machine breakdowns. Discrete Appl. Math. 110 (2001), 85-89.

[4] J. A. Aslam, A. Rasala, C. Stein, and N. E. Young. 1999. Improved bicriteria existence theorems for scheduling. In Proceedings of the Annual ACM-SIAM Symposium on Discrete Algorithms. 846-847.

[5] L. Chen, N. Megow, R. Rischke, and L. Stougie. 2015. Stochastic and robust scheduling in the cloud. In Proceedings of the International Conference on Approximation Algorithms for Combinatorial Optimization Problems and the International Conference on Randomization and Computation (APPROX-RANDOM'15). 175-186.

[6] Y. Disser, M. Klimm, N. Megow, and S. Stiller. 2014. Packing a knapsack of unknown capacity. In Proceedings of the Symposium on Theoretical Aspects of Computer Science. 276-287.

[7] L. Epstein, A. Levin, A. Marchetti-Spaccamela, N. Megow, J. Mestre, M. Skutella, and L. Stougie. 2012. Universal sequencing on an unreliable machine. SIAM f. Comput. 41 (2012), 565-586.

[8] R. L. Graham. 1966. Bounds for certain multiprocessing anomalies. Bell Syst. Techn. J. (1966), 1563-1581.

[9] R. Hassin and S. Rubinstein. 2002. Robust matchings. SIAM J. Discrete Math. 15 (2002), 530-537.

[10] K. Jansen, K. M. Klein, and J. Verschae. 2016. Closing the gap for makespan scheduling via sparsification technique. In Proceedings of the Automata, Languages, and Programming. 1-13.

[11] J. Matuschke, M. Skutella, and J. A. Soto. 2015. Robust randomized matchings. In Proceedings of the Annual ACM-SIAM Symposium on Discrete Algorithms. 1904-1915.

[12] N. Megow. 2015. Robustness and approximation for universal sequencing. Gems Combinat. Optim. Graph Alg. (2015), 133-141.

[13] J. Niño-Mora. 2009. Stochastic scheduling. In Encyclopedia of Optimization, C. A. Floudas and P. M. Pardalos (Eds.). Springer, New York, 3818-3824.

[14] L. K. Platzman and J. J. Bartholdi. 1989. Spacefilling curves and the planar travelling salesmanproblem. F. ACM 36 (1989), 719-737.

[15] A. Rasala, C. Stein, E. Torng, and P. Uthaisombut. 2002. Existence theorems, lower bounds and algorithms for scheduling to meet two objectives. In Proceedings of the Annual ACM-SIAM Symposium on Discrete Algorithms. 723-731.

[16] M. Schilling. 1992. Sequential partitioning. Amer. Math. Monthly 99 (1992), 846-855.

[17] D. B. Shmoys and M. Sozio. 2007. Approximation algorithms for 2-stage stochastic scheduling problems. In Proceedings of the Integer Programming and Combinatorial Optimization International Conference. 145-157.

Received April 2018; revised May 2019; accepted June 2019 